\title{
Adaptive Finite-Time Control for Spacecraft Rendezvous under Unknown System Parameters
}

\author{
Yuan Liu, ${ }^{1}$ Guojian Tang, ${ }^{1}$ Yuhang Li, ${ }^{2}$ Hang Li, ${ }^{1}$ Jing Ren, ${ }^{1}$ and Sai Zhang $\mathbb{D}^{3}$ \\ ${ }^{1}$ School of Astronautics, National University of Defense Technology, Changsha 410022, China \\ ${ }^{2}$ School of Electronic and Information Engineering, Northeast Agricultural University, Harbin 150030, China \\ ${ }^{3}$ College of Aerospace and Civil Engineering, Harbin Engineering University, Harbin 150001, China
}

Correspondence should be addressed to Sai Zhang; zhangs_22@hrbeu.edu.cn

Received 22 February 2020; Revised 23 June 2020; Accepted 11 July 2020; Published 5 August 2020

Academic Editor: Maj D. Mirmirani

Copyright ( 2020 Yuan Liu et al. This is an open access article distributed under the Creative Commons Attribution License, which permits unrestricted use, distribution, and reproduction in any medium, provided the original work is properly cited.

In this study, we investigated the sliding mode control (SMC) technology for the spacecraft rendezvous maneuver under unknown system parameters and external disturbance. With no knowledge of the mass and inertial matrix of the pursuer spacecraft, an adaptive SMC approach was devised using the hyperbolic tangent function to realize the control objective of reducing the chattering problem. In addition, the finite-time stability of the relative dynamics and the boundedness of the signals in the closed-loop system were derived under proposed method. The effectiveness and advantages of the proposed method were verified through theoretical analysis and numerical simulations.

\section{Introduction}

With rapid developments in the aerospace industry, spacecraft rendezvous technology has been extensively applied in space missions, such as deep space exploration, the establishment of space stations, and the detection of various components on Mars. Due to the fact that control systems are one of the most crucial technologies of the rendezvous maneuver, extensive attention of researchers has been attracted in the past decades. To guarantee the success of space missions, various methods are studied for spacecraft rendezvous maneuver. However, designing controllers for the spacecraft rendezvous maneuver is still a challenging task because of the strongly coupled nonlinear dynamics and unknown external environment. Current attitude controls of spacecraft include backstepping control $[1,2]$, adaptive control [3-5], and sliding mode control (SMC) [6-8].

However, the methods in [1-7] cannot be used in the spacecraft rendezvous maneuver, which severely limits their application. Considerable efforts are required during the controller design process in rendezvous missions to address the effects of complex external disturbance and coupled nonlin- ear dynamics. To reduce the effect of external disturbances, backstepping-based controllers, in which globally asymptotic stability can be achieved for closed-loop systems, have been investigated in detail in [9-11]. The common drawback in [9-11] is that the convergence rate of the control system is asymptotical, that is, the control objective can be achieved when time is infinite. To ensure finite-time convergence for the entire system, Wang et al. investigated control methods to accomplish the rendezvous mission by using the backstepping design [12]. However, actuator faults in actual spacecraft activities have not been considered in [9-12]. Unexpected and complex failures frequently occur in the actuators in spacecraft rendezvous missions. These failures result in the degradation of control performance. Furthermore, failures may occur during a mission in the event of limited communication bandwidth and transmission delays. To ensure mission success and avoid unexpected failures, adaptive SMC algorithms have been investigated to improve reliability $[13,14]$. Another aspect that deserves special attention is collision avoidance during the rendezvous maneuver, which has been ignored in previous articles. Artificial-potential-function-based SMC and backstepping 
control are proposed in $[15,16]$, respectively, for avoiding collisions during rendezvous maneuvers.

Results in [9-16] were obtained by regarding the inertial parameters as available valuables. However, inertial parameters are not always available to designers during real missions mainly because of fuel consumption in the pursuer spacecraft. Moreover, solar radiation pressure and disturbances in the space environment influence the inertial parameters. Considering these aspects, an adaptive SMC method was presented in [17] for controlling spacecraft maneuver under unknown inertia. The adaptive SMC of [17] was improved to an adaptive control algorithm based on gain and neural networks in [18] for addressing uncertainties and external disturbances. For spacecraft proximity operations with parametric uncertainties, an integrated adaptive backstepping control method and an adaptive control algorithm using dual quaternions were designed in $[19,20]$, respectively. However, the results from [17-20] cannot be directly applied to the spacecraft rendezvous maneuver. On the basis of [18-20], the uncertainties of the relative dynamics were compensated using robust adaptive backstepping control in [21] by introducing radial basis function neural networks. In contrast to [21], the problem of time-varying inertial parameters was studied in [22]. Similarly, the continuous adaptive control algorithm was combined with the projection algorithm in [23] for controlling the spacecraft rendezvous maneuver under time-varying inertia parameters and actuator faults.

The effective controllers in [17-23] that address uncertainties are asymptotically stable, that is, the system states converge to equilibrium when time is infinite. Unlike asymptotically stable controllers, finite-time control schemes have been widely studied and applied in spacecraft attitude control because of their high convergence rate and superior control performance $[8,24,25]$. Controllers for the unwinding phenomenon, which was not considered in [24, 25], are presented in [8]. Moreover, input saturation constraints, which influence the performance of spacecraft, were not considered in $[8,24,25]$. Therefore, an adaptive finite-time control algorithm was investigated in [26] to address the problem of unavoidable input saturations for spacecraft. As a continuation of [26], the collision problem between the pursuer spacecraft and the target spacecraft was studied using an adaptive finite-time antisaturation controller in [27].

Excellent finite-time stability can be achieved for the system by using the controllers in [8, 24-27]. However, the chattering phenomenon, which is the main cause of actuator damage, was not considered in these studies. A boundary layer function was incorporated into the controller in [28] to alleviate the chattering phenomenon. Similarly, continuous and chatter-free controllers were introduced in [29] for spacecraft rendezvous and docking. In this study, the finitetime control problem for spacecraft rendezvous was studied in terms of the existing chattering problem in the SMC and the unknown time-varying inertial parameters in real missions. The contributions of this paper are as follows:

(i) In contrast to the existing spacecraft control schemes for known inertia [10-13], unknown parameters are considered in this paper, which considerably extends the application of control methods for the spacecraft rendezvous maneuver

(ii) Unlike the controllers in [17-23], finite-time stability can be achieved for the system with a high convergence rate by using the proposed method even when the inertial parameters are unavailable to designers

(iii) The hyperbolic tangent function can be used in the control law to avoid the chattering problem. Furthermore, the sliding mode method was adopted in this study.

The remainder of this paper is arranged as follows. The dynamics model of the spacecraft is established in Section 2. The finite-time controller is described in Section 3. The effectiveness of the controller is proved through simulations in Section 4. Finally, the conclusion of this paper is presented in Section 5.

\section{Spacecraft Model and Preliminaries}

2.1. Relative Attitude Dynamic Model. The control equations for the attitude motion of a rigid spacecraft can be established by using the unit quaternion. According to [23], the rotation matrix $\boldsymbol{R} \in S O(3)$ and the unit quaternion $\mathbf{Q}=\left[q_{0}, \mathbf{q}^{\mathrm{T}}\right]^{\mathrm{T}} \in \Xi$ with $\Xi=\left\{\mathbf{Q} \in \mathbb{R} \times \mathbb{R}^{3 \times 3} \mid q_{0}^{2}+\mathbf{q}^{\mathrm{T}} \mathbf{q}=1\right\}$ are applied in attitude formulation. Furthermore, $\mathbf{Q}_{p}$ and $\mathbf{Q}_{t}$ represent the attitude of the pursuer and the target, respectively. Consequently, the relative attitude between the pursuer and the target can be expressed as follows:

$$
\tilde{\mathbf{Q}}=\left[\tilde{q}_{0}, \tilde{\mathbf{q}}^{\mathrm{T}}\right]^{\mathrm{T}}=\mathbf{Q}_{t}^{-1} \odot \mathbf{Q}_{p}
$$

The relative attitude kinematics model [25] can be expressed as follows:

$$
\begin{aligned}
& \dot{\tilde{q}}_{0}=-\frac{1}{2} \tilde{\boldsymbol{q}}_{v}{ }^{T} \tilde{\boldsymbol{\omega}}, \\
& \dot{\tilde{q}}_{v}=\frac{1}{2}\left(\tilde{\boldsymbol{q}}_{v}{ }^{\times}+\tilde{q}_{0} I_{3}\right) \tilde{\boldsymbol{\omega}},
\end{aligned}
$$

where $\tilde{\boldsymbol{\omega}}=\boldsymbol{\omega}_{p}-\tilde{\boldsymbol{R}} \boldsymbol{\omega}_{t}$ is the relative angular velocity, with $\boldsymbol{\omega}_{p}$ and $\omega_{t}$ denoting the angular velocity of the pursuer and target, respectively. For a vector $\boldsymbol{a}=\left[a_{1}, a_{2}, a_{3}\right]^{T}, \boldsymbol{a}^{x}$ can be defined using Eq. (3). The rotation matrix is defined in Eq. (4).

$$
\begin{aligned}
& \boldsymbol{a}^{x}=\left[\begin{array}{ccc}
0 & -a_{3} & a_{2} \\
a_{3} & 0 & -a_{1} \\
-a_{2} & a_{1} & 0
\end{array}\right], \\
& \tilde{\boldsymbol{R}} \triangleq \boldsymbol{R}(\tilde{\boldsymbol{q}})=\left(\tilde{q}_{0}^{2}-\tilde{\boldsymbol{q}}_{v}^{T} \tilde{\boldsymbol{q}}_{v}\right) \boldsymbol{I}_{3}+2 \tilde{\boldsymbol{q}}_{v} \tilde{\boldsymbol{q}}_{v}^{T}-2 \tilde{\boldsymbol{q}}_{0} \tilde{\boldsymbol{q}}_{v}^{x} .
\end{aligned}
$$


According to the Euler-Newton formulas of the pursuer and target [27], the corresponding attitude dynamics can be expressed as follows:

$$
\begin{aligned}
& \mathbf{J}_{t} \dot{\boldsymbol{\omega}}_{t}+\boldsymbol{\omega}_{t} \times \mathbf{J}_{t} \boldsymbol{\omega}_{t}=0, \\
& \mathbf{J} \dot{\boldsymbol{\omega}}_{p}+\boldsymbol{\omega}_{p}{ }^{\times} \mathbf{J} \boldsymbol{\omega}_{p}=\boldsymbol{\tau}+\boldsymbol{\tau}_{d},
\end{aligned}
$$

where $\mathbf{J}_{t} \in \mathrm{R}^{3 \times 3}$ and $\mathbf{J} \in \mathrm{R}^{3 \times 3}$ denote the inertial matrices and $\tau \in \mathrm{R}^{3}$ and $\tau_{d} \in \mathrm{R}^{3}$ represent the control and external disturbance torques, respectively.

The derivative of $\tilde{\boldsymbol{\omega}}$ satisfies the following expression:

$$
\dot{\tilde{\boldsymbol{\omega}}}=\dot{\boldsymbol{\omega}}_{p}-\dot{\tilde{\mathbf{R}}} \boldsymbol{\omega}_{t}-\tilde{\mathbf{R}} \dot{\boldsymbol{\omega}}_{t}
$$

Combining Eqs. (5)-(7) and considering that $\dot{\tilde{\mathbf{R}}}=\tilde{\mathbf{R}} \tilde{\boldsymbol{\omega}}^{\times}$, the following equation is obtained:

$$
\mathbf{J} \dot{\tilde{\omega}}=-\mathbf{C}_{r} \tilde{\boldsymbol{\omega}}-\mathbf{n}_{r}+\boldsymbol{\tau}+\boldsymbol{\tau}_{d},
$$

where $\mathbf{C}_{r}=\mathbf{J}\left(\tilde{\mathbf{R}} \boldsymbol{\omega}_{t}\right)^{\times}+\left(\tilde{\mathbf{R}} \boldsymbol{\omega}_{t}\right)^{\times} \mathbf{J}-\left(\mathbf{J}\left(\tilde{\boldsymbol{\omega}}+\tilde{\mathbf{R}} \boldsymbol{\omega}_{t}\right)\right)^{\times}$and $\mathbf{n}_{r}=$ $\left(\tilde{\mathbf{R}} \boldsymbol{\omega}_{t}\right)^{\times} \mathbf{J} \tilde{\mathbf{R}} \boldsymbol{\omega}_{t}+\mathbf{J} \tilde{\mathbf{R}} \dot{\boldsymbol{\omega}}_{t}$.

2.2. Relative Orbit Dynamics Model. According to the theory of relative motion, $\mathbf{r}_{\mathbf{p}}$ and $\boldsymbol{v}_{\mathbf{p}}$ are used to express the position and velocity of the pursuer, as presented in Eqs. (9) and (10), respectively.

$$
\begin{aligned}
& \mathbf{r}_{\mathbf{p}}=\tilde{\mathbf{r}}+\tilde{\mathbf{R}}\left(\mathbf{r}_{t}+\boldsymbol{\sigma}_{t}\right), \\
& \boldsymbol{v}_{\mathbf{p}}=\tilde{\boldsymbol{v}}+\tilde{\mathbf{R}}\left(\boldsymbol{v}_{t}+\boldsymbol{\omega}_{t}^{\times} \boldsymbol{\sigma}_{t}\right),
\end{aligned}
$$

where $\mathbf{r}_{t}$ and $\boldsymbol{v}_{t}$ are the position and velocity of the target, respectively; $\tilde{\mathbf{r}}$ and $\tilde{\mathbf{v}}$ are the relative position and relative velocity, respectively; and the constant vector $\boldsymbol{\sigma}_{t} \in R^{3}$ denotes the desired rendezvous position. The derivative of Eq. (9) can be expressed as follows:

$$
\dot{\mathbf{r}}_{\mathbf{p}}=\dot{\tilde{\mathbf{r}}}+\dot{\tilde{\mathbf{R}}}\left(\mathbf{r}_{t}+\sigma_{t}\right)+\tilde{\mathbf{R}} \dot{\mathbf{r}}_{t}
$$

According to the description of kinematic principles in [30], the following equations are obtained:

$$
\begin{aligned}
& \dot{\mathbf{r}}_{t}=\boldsymbol{v}_{t}-\boldsymbol{\omega}_{t}^{\times} \mathbf{r}_{t}, \\
& \dot{\mathbf{r}}_{\mathbf{p}}=\boldsymbol{v}_{\mathbf{p}}-\boldsymbol{\omega}_{p}^{\times} \mathbf{r}_{\mathbf{p}} .
\end{aligned}
$$

By combining Eqs. (11) and (13), the following expression is obtained:

$$
\dot{\tilde{\mathbf{r}}}+\dot{\tilde{\mathbf{R}}}\left(\mathbf{r}_{t}+\boldsymbol{\sigma}_{t}\right)+\tilde{\mathbf{R}} \dot{\mathbf{r}}_{t}=\boldsymbol{v}_{\mathbf{p}}-\boldsymbol{\omega}_{p}^{\times} \mathbf{r}_{\mathbf{p}} .
$$

From the aforementioned equation, the following expression is obtained:

$$
\dot{\tilde{\mathbf{r}}}=\tilde{\mathbf{v}}-\mathbf{C}_{t} \tilde{\mathbf{r}},
$$

where $\mathbf{C}_{t}=\left(\tilde{\boldsymbol{\omega}}+\tilde{\mathbf{R}} \boldsymbol{\omega}_{t}\right)^{\times}$. Consequently, the derivative of $\boldsymbol{v}_{\mathbf{p}}$ is expressed as follows:

$$
\dot{\boldsymbol{v}}_{\mathbf{p}}=\dot{\tilde{\mathbf{v}}}+\dot{\tilde{\mathbf{R}}}\left(\boldsymbol{v}_{t}+\boldsymbol{\omega}_{t}^{\times} \boldsymbol{\sigma}_{t}\right)+\tilde{\mathbf{R}}\left(\dot{\boldsymbol{v}}_{t}+\dot{\boldsymbol{\omega}}_{t}^{\times} \boldsymbol{\sigma}_{t}\right) .
$$

According to the theory in [30], Eqs. (17) and (18) define the position dynamics of spacecraft.

$$
\begin{aligned}
& m_{t} \dot{\boldsymbol{v}}_{t}+m_{t} \boldsymbol{\omega}_{t}^{\times} \boldsymbol{v}_{t}=0, \\
& m_{p} \dot{\boldsymbol{v}}_{p}+m_{p} \boldsymbol{\omega}_{p}^{\times} \boldsymbol{v}_{p}=\mathbf{f}+\mathbf{f}_{d},
\end{aligned}
$$

where $m_{t}$ and $m_{p}$ are constants that define the masses of the target and purser, respectively, and $\mathbf{f} \in \mathrm{R}^{N}$ and $\mathbf{f}_{d} \in \mathrm{R}^{3}$ denote the control and external disturbance forces, respectively. From Eqs. (16) and (18), the following equation can be obtained [23]:

$$
m_{p}\left[\dot{\tilde{\mathbf{v}}}+\dot{\tilde{\mathbf{R}}}\left(\mathbf{v}_{t}+\boldsymbol{\omega}_{t}^{\times} \boldsymbol{\sigma}_{t}\right)+\tilde{\mathbf{R}}\left(\dot{\mathbf{v}}_{t}+\dot{\boldsymbol{\omega}}_{t}^{\times} \boldsymbol{\sigma}_{t}\right)\right]+m_{p} \boldsymbol{\omega}_{p}^{\times} \boldsymbol{v}_{p}=\mathbf{f}+\mathbf{f}_{d} .
$$

Thus, the following equation is obtained:

$$
m_{p} \dot{\tilde{\mathbf{v}}}=-m_{p} \mathbf{C}_{t} \tilde{\mathbf{v}}-m_{p} \mathbf{n}_{t}+\mathbf{f}+\mathbf{f}_{d},
$$

where $\mathbf{n}_{t}=\left(\tilde{\mathbf{R}} \boldsymbol{\omega}_{t}\right)^{\times} \tilde{\mathbf{R}} \mathbf{v}_{t}+\tilde{\mathbf{R}} \dot{\mathbf{v}}_{t}+\tilde{\boldsymbol{\omega}}{ }^{\times} \tilde{\mathbf{R}} \boldsymbol{\sigma}_{t}^{\times} \boldsymbol{\omega}_{t}-\tilde{\mathbf{R}} \boldsymbol{\sigma}_{t}^{\times} \dot{\boldsymbol{\omega}}_{t}$.

For convenience, the control torques can be expressed as follows:

$$
\boldsymbol{\tau}=\boldsymbol{\varepsilon}_{r}, \mathbf{f}=\boldsymbol{\varepsilon}_{t}
$$

where $\boldsymbol{\varepsilon}_{r} \in \mathrm{R}^{N}$ and $\boldsymbol{\varepsilon}_{t} \in \mathrm{R}^{N}$. By combining Eqs. (8), (20), and (21), the relative dynamics can be established as follows:

$$
\begin{gathered}
\boldsymbol{J} \dot{\tilde{\boldsymbol{\omega}}}=-\mathbf{C}_{r} \tilde{\boldsymbol{\omega}}-\mathbf{n}_{r}+\boldsymbol{\varepsilon}_{r}+\mathbf{d}_{r}, \\
m \dot{\tilde{\boldsymbol{v}}}=-m \mathbf{C}_{t} \tilde{\boldsymbol{v}}-m \mathbf{n}_{t}+\boldsymbol{\varepsilon}_{t}+\mathbf{d}_{t},
\end{gathered}
$$

where $\mathbf{d}_{r}=\boldsymbol{\tau}_{d}$ and $\mathbf{d}_{t}=\mathbf{f}_{d}$.

During the controller design process, the exact motion information of the target spacecraft is assumed to be available to the tracker spacecraft. In this paper, we focus on designing controllers for the dynamics expressed in Eqs. (21) and (22) to ensure the finite-time stability of the closed-loop system even in the presence of unknown inertial parameters and external disturbance.

To facilitate the controller design, the following assumptions were made:

Assumption 1. The dynamics of the target are stable, which implies that $\boldsymbol{\omega}_{t}, \dot{\boldsymbol{\omega}}_{t}, \mathbf{v}_{t}$, and $\dot{\mathbf{v}}_{t}$ are bounded and satisfy $\left\|\boldsymbol{\omega}_{t}\right\|$ $\leq a_{1},\left\|\dot{\boldsymbol{\omega}}_{t}\right\| \leq a_{2}$, and $\left\|\dot{\mathbf{v}}_{t}\right\| \leq a_{4}$, where $a_{1}, a_{2}, a_{3}$, and $a_{4}$ are positive constants.

Assumption 2. The inertial matrix $\mathbf{J}$ and the mass of the pursuer $m$ are unknown and satisfy $\lambda_{1} \mathbf{I}^{3 \times 3} \leq \mathbf{J} \leq \lambda_{2} \mathbf{I}^{3 \times 3}$ and $m \leq \lambda_{3}$, where $\lambda_{i}(i=1,2,3)$ are unknown positive constants. 
Assumption 3. The external disturbance $\mathbf{d}_{r}$ and $\mathbf{d}_{t}$ are unknown bounded vectors, which satisfy $\left\|\mathbf{d}_{r}\right\| \leq D_{r}<c_{1}$ and $\left\|\mathbf{d}_{t}\right\| \leq D_{t}<c_{2}$, where $c_{1}$ and $c_{2}$ are positive constants.

\subsection{Preliminaries}

Lemma 4 (see [24]). For an arbitrary real number $x \in R$, $\mu>0$, and $\kappa=0.2785$, the following relation exists:

$$
0<|x|-x \tanh (\mu x) \leq \frac{\kappa}{\mu} .
$$

Lemma 5 (see [26]). For the system expressed in Eqs. (21) and (22), if the Lyapunov function $V_{1}$ exists, it satisfies the following expression:

$$
\dot{V}_{1} \leq-\alpha V_{1}^{p}+\sigma
$$

where $\alpha>0,0<p<1$, and $0<\sigma<\infty$, and the system converges to a region in finite time.

\section{Attitude Controller Design}

3.1. Basic Controller Design. To complete the design of the attitude and orbit control schemes, two sliding mode variables are defined as follows:

$$
\begin{aligned}
& \mathbf{s}_{1}=\tilde{\boldsymbol{\omega}}+k_{1} \tilde{\mathbf{q}}_{\mathrm{v}}+k_{2} \tanh \left(\tilde{\mathbf{q}}_{\mathrm{v}}\right), \\
& \mathbf{s}_{2}=\tilde{\mathbf{v}}+k_{3} \tilde{\mathbf{r}}+k_{4} \tanh (\tilde{\mathbf{r}}),
\end{aligned}
$$

where $k_{1}, k_{2}, k_{3}$, and $k_{4}$ are positive constants.

The derivatives of $\mathbf{s}_{1}$ and $\mathbf{s}_{2}$ are expressed as follows:

$$
\begin{aligned}
\mathbf{J} \dot{\mathbf{s}}_{1}= & \boldsymbol{J} \dot{\tilde{\boldsymbol{\omega}}}+k_{1} J \dot{\tilde{\mathbf{q}}}_{v}+k_{2} \mathbf{J}\left(1+\tanh ^{\mathrm{T}}\left(\tilde{\mathbf{q}}_{v}\right) \tanh \left(\tilde{\mathbf{q}}_{v}\right)\right) \dot{\tilde{\mathbf{q}}}_{v} \\
= & -\mathbf{C}_{r} \tilde{\boldsymbol{\omega}}-\mathbf{n}_{r}+\boldsymbol{\varepsilon}_{r}+\mathbf{d}_{r} \\
& +\frac{1}{2} \boldsymbol{J}\left[k_{1}+k_{2}\left(1+\tanh ^{\mathrm{T}}\left(\tilde{\mathbf{q}}_{v}\right) \tanh \left(\tilde{\mathbf{q}}_{v}\right)\right)\right] \\
& \cdot\left(\tilde{\mathbf{q}}_{v}^{\times}+\tilde{q}_{0} \mathbf{I}_{3}\right) \tilde{\boldsymbol{\omega}} \\
m \dot{\mathbf{s}}_{2}= & m \dot{\tilde{\mathbf{v}}}+m k_{3} \dot{\tilde{\mathbf{r}}}+m k_{4}\left(1+\tanh ^{\mathrm{T}}(\tilde{\mathbf{r}}) \tanh (\tilde{\mathbf{r}})\right) \dot{\tilde{\mathbf{r}}} \\
= & -m \mathbf{C}_{t} \tilde{\mathbf{v}}-m \mathbf{n}_{t}+\boldsymbol{\varepsilon}_{t}+\mathbf{d}_{t} \\
& +m\left[k_{3}+k_{4}\left(1+\tanh ^{\mathrm{T}}(\tilde{\mathbf{r}}) \tanh (\tilde{\mathbf{r}})\right)\right]\left(\tilde{\mathbf{v}}-\mathbf{C}_{t} \tilde{\mathbf{r}}\right) .
\end{aligned}
$$

Considering the aforementioned assumptions and the relations $\left\|\tilde{\mathbf{q}}_{v}^{\times}+\tilde{q}_{0} \mathbf{I}_{3}\right\|=1, \quad\left\|\tanh ^{\mathrm{T}}\left(\tilde{\mathbf{q}}_{v}\right) \tanh \left(\tilde{\mathbf{q}}_{v}\right)\right\| \leq 1, \quad \| 1+$ $\tanh ^{\mathrm{T}}(\tilde{\mathbf{r}}) \tanh (\tilde{\mathbf{r}}) \| \leq 1$, and $\tilde{\mathbf{q}}_{v}^{T} \tilde{\mathbf{q}}_{v}+\tilde{q}_{0}^{2}=1$, the following conditions can be derived:

$$
\begin{array}{r}
\left\|-\mathbf{C}_{r} \tilde{\boldsymbol{\omega}}-\mathbf{n}_{r}+\frac{1}{2} \boldsymbol{J}\left[k_{1}+k_{2}\left(1+\tanh ^{\mathrm{T}}\left(\tilde{\mathbf{q}}_{v}\right) \tanh \left(\tilde{\mathbf{q}}_{v}\right)\right)\right]\left(\tilde{\mathbf{q}}_{v}^{\times}+\tilde{q}_{0} \mathbf{I}_{3}\right) \tilde{\boldsymbol{\omega}}\right\| \leq \alpha_{1} \varsigma_{1}, \\
\left\|-m \mathbf{C}_{t} \tilde{\boldsymbol{v}}-m \mathbf{n}_{t}+m\left[k_{3}+k_{4}\left(1+\tanh ^{\mathrm{T}}(\tilde{\mathbf{r}}) \tanh (\tilde{\mathbf{r}})\right)\right]\left(\tilde{\boldsymbol{v}}-\mathbf{C}_{t} \tilde{\mathbf{r}}\right)\right\| \leq \alpha_{2} \varsigma_{2},
\end{array}
$$

where $\alpha_{1}$ and $\alpha_{2}$ are unknown positive constants, $\varsigma_{1}=$ $\left\|\psi_{r}\right\|, \quad \varsigma_{2}=\left\|\psi_{t}\right\|, \quad \psi_{r}=\left[\|\tilde{\boldsymbol{\omega}}\|^{2},\|\tilde{\boldsymbol{\omega}}\|, 1\right]^{\mathrm{T}}, \quad$ and $\quad \psi_{t}=$ $[\|\tilde{\boldsymbol{\omega}}\|\|\tilde{\mathbf{v}}\|,\|\tilde{\boldsymbol{\omega}}\|\|\tilde{\mathbf{r}}\|,\|\tilde{\boldsymbol{\omega}}\|,\|\tilde{\mathbf{v}}\|,\|\tilde{\mathbf{r}}\|, 1]^{\mathrm{T}}$. Control laws for the relative attitude and orbit dynamics can be designed as follows:

$\boldsymbol{\varepsilon}_{r}=-k_{5} \tanh \left(\mathbf{s}_{1}\right)-k_{6} \mathbf{s}_{1}-\widehat{D}_{r} \tanh \left(\frac{\mathbf{s}_{1}}{\mu_{1}}\right)-\widehat{\alpha}_{1} \varsigma_{1} \tanh \left(\frac{\varsigma_{1} \mathbf{s}_{1}}{\mu_{3}}\right)$

$\boldsymbol{\varepsilon}_{t}=-k_{7} \tanh \left(\mathbf{s}_{2}\right)-k_{8} \mathbf{s}_{2}-\widehat{D}_{t} \tanh \left(\frac{\mathbf{s}_{2}}{\mu_{2}}\right)-\widehat{\alpha}_{2} \varsigma_{2} \tanh \left(\frac{\varsigma_{2} \mathbf{s}_{2}}{\mu_{4}}\right)$,

where $k_{i}>0, i=5,6,7,8$ and $\mu_{i}>0, i=1,2,3,4$. In the aforementioned equation, $\widehat{D}_{r}, \widehat{D}_{t}, \widehat{\alpha}_{1}$, and $\widehat{\alpha}_{2}$ are the estimations of $D_{r}, D_{t}, \alpha_{1}$, and $\alpha_{2}$, respectively. The terms $\dot{\hat{D}}_{r}, \dot{\hat{D}}_{t}, \widehat{\alpha}_{1}$, and $\widehat{\alpha}_{2}$ are defined as follows:

$$
\begin{aligned}
& \dot{\hat{D}}_{r}=c_{1}\left(\left\|\mathbf{s}_{1}\right\| \tanh \left(\frac{\left\|\mathbf{s}_{1}\right\|}{3 \mu_{1}}\right)-c_{5} \widehat{D}_{r}\right), \\
& \dot{\hat{D}}_{t}=c_{2}\left(\left\|\mathbf{s}_{2}\right\| \tanh \left(\frac{\left\|\mathbf{s}_{2}\right\|}{3 \mu_{2}}\right)-c_{6} \widehat{D}_{t}\right), \\
& \dot{\hat{\alpha}}_{1}=c_{3}\left(\varsigma_{1}\left\|\mathbf{s}_{1}\right\| \tanh \left(\frac{s_{1}\left\|\mathbf{s}_{1}\right\|}{3 \mu_{3}}\right)-c_{7} \widehat{\alpha}_{1}\right), \\
& \dot{\hat{\alpha}}_{2}=c_{4}\left(\varsigma_{2}\left\|\mathbf{s}_{2}\right\| \tanh \left(\frac{\varsigma_{2}\left\|\mathbf{s}_{2}\right\|}{3 \mu_{4}}\right)-c_{8} \widehat{\alpha}_{2}\right),
\end{aligned}
$$

where $c_{i}>0, i=1,2, \cdots, 8$. The estimation errors are defined as follows:

$$
\begin{aligned}
& \tilde{D}_{r}=D_{r}-\widehat{D}_{r}, \\
& \dot{\tilde{D}}_{t}=D_{t}-\widehat{D}_{t}, \\
& \tilde{\alpha}_{1}=\alpha_{1}-\widehat{\alpha}_{1}, \\
& \tilde{\alpha}_{2}=\alpha_{2}-\widehat{\alpha}_{2} .
\end{aligned}
$$

Theorem 6. For the dynamics expressed in Eqs. (22) and (23) with unknown system parameters $\mathbf{J}$ and $m$, the finite-time stability of the system can be achieved using the controller proposed in Eqs. (29)-(34).

Proof. To prove the stability of the system, the Lyapunov function is designed as follows:

$$
V_{1}=\frac{1}{2} \mathbf{s}_{1}^{\mathrm{T}} \mathbf{J} \mathbf{s}_{1}+\frac{1}{2} \mathbf{s}_{2}^{\mathrm{T}} m \mathbf{s}_{2}+\frac{1}{2 c_{1}} \tilde{D}_{r}^{2}+\frac{1}{2 c_{2}} \tilde{D}_{t}^{2}+\frac{1}{2 c_{3}} \alpha_{1}^{2}+\frac{1}{2 c_{4}} \alpha_{2}^{2} .
$$

According to the relative system dynamics and control laws, the following expressions are obtained: 


$$
\begin{aligned}
\dot{V}_{1}= & \mathbf{s}_{1}^{\mathrm{T}} \mathbf{J} \dot{\mathbf{s}}_{1}+\mathbf{s}_{2}^{\mathrm{T}} m \dot{\mathbf{s}}_{2}+\frac{1}{c_{1}} \tilde{D}_{r} \dot{\tilde{D}}_{r}+\frac{1}{c_{2}} \tilde{D}_{t} \dot{\tilde{D}}_{t}+\frac{1}{c_{3}} \tilde{\alpha}_{1} \dot{\tilde{\alpha}}_{1}+\frac{1}{c_{4}} \tilde{\alpha}_{2} \dot{\tilde{\alpha}}_{2} \\
= & \mathbf{s}_{1}^{\mathrm{T}} \mathbf{J} \dot{\mathbf{s}}_{1}+\mathbf{s}_{2}^{\mathrm{T}} m \dot{\mathbf{s}}_{2}-\frac{1}{c_{1}} \tilde{D}_{r} \dot{\hat{D}}_{r}-\frac{1}{c_{2}} \tilde{D}_{t} \dot{\hat{D}}_{t}-\frac{1}{c_{3}} \tilde{\alpha}_{1} \dot{\hat{\alpha}}_{1}-\frac{1}{c_{4}} \tilde{\alpha}_{2} \dot{\hat{\alpha}}_{2} \\
= & \mathbf{s}_{1}^{\mathrm{T}}\left[-\mathbf{C}_{r} \tilde{\boldsymbol{\omega}}-\mathbf{n}_{r}+\boldsymbol{\varepsilon}_{r}+\mathbf{d}_{r}\right. \\
& \left.+\frac{1}{2} \boldsymbol{J}\left[k_{1}+k_{2}\left(1+\tanh ^{\mathrm{T}}\left(\tilde{\mathbf{q}}_{v}\right) \tanh \left(\tilde{\mathbf{q}}_{v}\right)\right)\right]\left(\tilde{\mathbf{q}}_{v}{ }^{\times}+\tilde{q}_{0} \mathbf{I}_{3}\right) \tilde{\boldsymbol{\omega}}\right] \\
& +\mathbf{s}_{2}^{\mathrm{T}}\left[-m \mathbf{C}_{t} \tilde{\boldsymbol{v}}-m \mathbf{n}_{t}+\boldsymbol{\varepsilon}_{t}+\mathbf{d}_{t}\right. \\
& +m\left[k_{3}+k_{4}\left(1+\tanh ^{\mathrm{T}}\left(\tilde{\mathbf{r}}_{)} \tanh (\tilde{\mathbf{r}})\right)\right]\left(\tilde{\mathbf{v}}-\mathbf{C}_{t} \tilde{\mathbf{r}}\right)\right] \\
& -\frac{1}{c_{1}} \tilde{D}_{r} \dot{\hat{D}}_{r}-\frac{1}{c_{2}} \tilde{D}_{t} \dot{\hat{D}}_{t}-\frac{1}{c_{3}} \tilde{\alpha}_{1} \dot{\hat{\alpha}}_{1}-\frac{1}{c_{4}} \tilde{\alpha}_{2} \dot{\hat{\alpha}}_{2} .
\end{aligned}
$$

Then, the following equations are derived:

$$
\begin{aligned}
& \mathbf{s}_{1}^{\mathrm{T}}\left[-\mathbf{C}_{r} \tilde{\boldsymbol{\omega}}-\mathbf{n}_{r}+\frac{1}{2} \boldsymbol{J}\left[k_{1}+k_{2}\left(1+\tanh ^{\mathrm{T}}\left(\tilde{\mathbf{q}}_{v}\right) \tanh \left(\tilde{\mathbf{q}}_{v}\right)\right)\right]\left(\tilde{\mathbf{q}}_{v}{ }^{\times}+\tilde{q}_{0} \mathbf{I}_{3}\right) \tilde{\boldsymbol{\omega}}\right] \\
& \quad \leq \alpha_{1} \varsigma_{1}\left\|\mathbf{s}_{1}\right\|,
\end{aligned}
$$

$$
\begin{aligned}
& \mathbf{s}_{2}^{\mathrm{T}}\left[-m \mathbf{C}_{t} \tilde{\boldsymbol{v}}-m \mathbf{n}_{t}+m\left[k_{3}+k_{4}\left(1+\tanh ^{\mathrm{T}}(\tilde{\mathbf{r}}) \tanh (\tilde{\mathbf{r}})\right)\right]\left(\tilde{\boldsymbol{v}}-\mathbf{C}_{t} \tilde{\mathbf{r}}\right)\right] \\
& \leq \alpha_{2} \varsigma_{2}\left\|\mathbf{s}_{2}\right\| .
\end{aligned}
$$

The following equations are obtained from Eqs. (37)-(39):

$$
\begin{gathered}
\dot{V}_{1} \leq \alpha_{1} \varsigma_{1}\left\|\mathbf{s}_{1}\right\|+\alpha_{2} \varsigma_{2}\left\|\mathbf{s}_{2}\right\|+\mathbf{s}_{1}^{\mathrm{T}}\left(\boldsymbol{\varepsilon}_{r}+\mathbf{d}_{r}\right)+\mathbf{s}_{2}^{\mathrm{T}}\left(\boldsymbol{\varepsilon}_{t}+\mathbf{d}_{t}\right) \\
-\frac{1}{c_{1}} \tilde{D}_{r} \dot{\hat{D}}_{r}-\frac{1}{c_{2}} \tilde{D}_{t} \dot{\hat{D}}_{t}-\frac{1}{c_{3}} \tilde{\alpha}_{1} \dot{\widehat{\alpha}}_{1}-\frac{1}{c_{4}} \tilde{\alpha}_{2} \dot{\widehat{\alpha}}_{2} .
\end{gathered}
$$

Substituting the equations of the proposed controllers into Eq. (40), we obtain the following expression:

$$
\begin{aligned}
\dot{V}_{1} \leq & \alpha_{1} \varsigma_{1}\left\|\mathbf{s}_{1}\right\|+\alpha_{2} \varsigma_{2}\left\|\mathbf{s}_{2}\right\|+\mathbf{s}_{1}^{\mathrm{T}} \mathbf{d}_{r}+\mathbf{s}_{2}^{\mathrm{T}} \mathbf{d}_{t} \\
& +\mathbf{s}_{1}^{\mathrm{T}}\left(-k_{5} \tanh \left(\mathbf{s}_{1}\right)-k_{6} \mathbf{s}_{1}-\widehat{D}_{r} \tanh \left(\frac{\mathbf{s}_{1}}{\mu_{1}}\right)\right. \\
& \left.-\widehat{\alpha}_{1} \varsigma_{1} \tanh \left(\frac{\varsigma_{1} \mathbf{s}_{1}}{\mu_{3}}\right)\right)+\mathbf{s}_{2}^{\mathrm{T}}\left(-k_{7} \tanh \left(\mathbf{s}_{2}\right)\right. \\
& \left.-k_{8} \mathbf{s}_{2}-\widehat{D}_{t} \tanh \left(\frac{\mathbf{s}_{2}}{\mu_{2}}\right)-\widehat{\alpha}_{2} \varsigma_{2} \tanh \left(\frac{\varsigma_{2} \mathbf{s}_{2}}{\mu_{4}}\right)\right) \\
& -\frac{1}{c_{1}} \tilde{D}_{r} \dot{\hat{D}}_{r}-\frac{1}{c_{2}} \tilde{D}_{t} \dot{\hat{D}}_{t}-\frac{1}{c_{3}} \tilde{\alpha}_{1} \dot{\hat{\alpha}}_{1}-\frac{1}{c_{4}} \tilde{\alpha}_{2} \dot{\hat{\alpha}}_{2} \\
\leq & \alpha_{1} \varsigma_{1}\left\|\mathbf{s}_{1}\right\|+\alpha_{2} \varsigma_{2}\left\|\mathbf{s}_{2}\right\|+\left\|\mathbf{s}_{1}\right\| D_{r}+\left\|\mathbf{s}_{2}\right\| D_{t}-k_{5}\left\|\mathbf{s}_{1}\right\| \\
& -k_{6}\left\|\mathbf{s}_{1}\right\|^{2}-\mathbf{s}_{1}^{\mathrm{T}} \widehat{D}_{r} \tanh \left(\frac{\mathbf{s}_{1}}{\mu_{1}}\right)-\mathbf{s}_{1}^{\mathrm{T}} \widehat{\alpha}_{1} \varsigma_{1} \tanh \left(\frac{\varsigma_{1} \mathbf{s}_{1}}{\mu_{3}}\right) \\
& -k_{7}\left\|\mathbf{s}_{2}\right\|-k_{8}\left\|\mathbf{s}_{2}\right\|^{2}-\mathbf{s}_{2}^{\mathrm{T}} \widehat{D}_{t} \tanh \left(\frac{\mathbf{s}_{2}}{\mu_{2}}\right) \\
& -\mathbf{s}_{2}^{\mathrm{T}} \widehat{\alpha}_{2} \varsigma_{2} \tanh \left(\frac{\varsigma_{2} \mathbf{s}_{2}}{\mu_{4}}\right)-\frac{1}{c_{1}} \tilde{D}_{r} \dot{\hat{D}}_{r}-\frac{1}{c_{2}} \tilde{D}_{t} \dot{\hat{D}}_{t} \\
& -\frac{1}{c_{3}} \tilde{\alpha}_{1} \dot{\hat{\alpha}}_{1}-\frac{1}{c_{4}} \tilde{\alpha}_{2} \dot{\hat{\alpha}}_{2}+3 \kappa\left(k_{5}+k_{7}\right) .
\end{aligned}
$$

From Lemma 4, we obtain the following condition:

$$
\begin{gathered}
-\widehat{D}_{r} \mathbf{s}_{1}^{\mathrm{T}} \tanh \left(\frac{\mathbf{s}_{1}}{\mu_{1}}\right) \leq-\left\|\mathbf{s}_{1}\right\| \widehat{D}_{r}+3 \widehat{D}_{r} \mu_{1} \kappa, \\
-\widehat{D}_{t} \mathbf{s}_{2}^{\mathrm{T}} \tanh \left(\frac{\mathbf{s}_{2}}{\mu_{2}}\right) \leq-\left\|\mathbf{s}_{2}\right\| \widehat{D}_{t}+3 \widehat{D}_{t} \mu_{2} \kappa, \\
-\varsigma_{1} \widehat{\alpha}_{1} \mathbf{s}_{1}^{\mathrm{T}} \tanh \left(\frac{\varsigma_{1} \mathbf{s}_{1}}{\mu_{3}}\right) \leq-\varsigma_{1} \widehat{\alpha}_{1}\left\|\mathbf{s}_{1}\right\|+3 \widehat{\alpha}_{1} \mu_{3} \kappa, \\
-\varsigma_{2} \widehat{\alpha}_{2} \mathbf{s}_{2}^{\mathrm{T}} \tanh \left(\frac{\varsigma_{2} \mathbf{s}_{2}}{\mu_{4}}\right) \leq-\varsigma_{2} \widehat{\alpha}_{2}\left\|\mathbf{s}_{2}\right\|+3 \widehat{\alpha}_{2} \mu_{4} \kappa .
\end{gathered}
$$

Consequently, Eq. (41) can be rewritten as follows:

$$
\begin{aligned}
\dot{V}_{1} \leq & \alpha_{1} \varsigma_{1}\left\|\mathbf{s}_{1}\right\|+\alpha_{2} \varsigma_{2}\left\|\mathbf{s}_{2}\right\|+\left\|\mathbf{s}_{1}\right\| D_{r}+\left\|\mathbf{s}_{2}\right\| D_{t}-k_{5}\left\|\mathbf{s}_{1}\right\| \\
& -k_{6}\left\|\mathbf{s}_{1}\right\|^{2}-\left\|\mathbf{s}_{1}\right\| \widehat{D}_{r}+3 \widehat{D}_{r} \mu_{1} \kappa-\widehat{\alpha}_{1} \varsigma_{1}\left\|\mathbf{s}_{1}\right\|+3 \widehat{\alpha}_{1} \varsigma_{1} \mu_{3} \kappa \\
& -k_{7}\left\|\mathbf{s}_{2}\right\|-k_{8}\left\|\mathbf{s}_{2}\right\|^{2}-\left\|\mathbf{s}_{2}\right\| \widehat{D}_{t}+3 \widehat{D}_{t} \mu_{2} \kappa-\widehat{\alpha}_{2} \varsigma_{2}\left\|\mathbf{s}_{2}\right\| \\
& +3 \widehat{\alpha}_{2} \varsigma_{2} \mu_{4} \kappa-\frac{1}{c_{1}} \tilde{D}_{r} \dot{\hat{D}}_{r}-\frac{1}{c_{2}} \tilde{D}_{t} \dot{\hat{D}}_{t}-\frac{1}{c_{3}} \tilde{\alpha}_{1} \dot{\widehat{\alpha}}_{1}-\frac{1}{c_{4}} \tilde{\alpha}_{2} \dot{\hat{\alpha}}_{2} \\
& +3 \kappa\left(k_{5}+k_{7}\right)=\tilde{\alpha}_{1} \varsigma_{1}\left\|\mathbf{s}_{1}\right\|+\tilde{\alpha}_{2} \varsigma_{2}\left\|\mathbf{s}_{2}\right\|+\left\|\mathbf{s}_{1}\right\| \tilde{D}_{r} \\
& +\left\|\mathbf{s}_{2}\right\| \tilde{D}_{t}-k_{5}\left\|\mathbf{s}_{1}\right\|-k_{6}\left\|\mathbf{s}_{1}\right\|^{2}-k_{7}\left\|\mathbf{s}_{2}\right\|-k_{8}\left\|\mathbf{s}_{2}\right\|^{2} \\
& -\frac{1}{c_{1}} \tilde{D}_{r} \dot{\hat{D}}_{r}-\frac{1}{c_{2}} \tilde{D}_{t} \dot{\hat{D}}_{t}-\frac{1}{c_{3}} \tilde{\alpha}_{1} \dot{\hat{\alpha}}_{1}-\frac{1}{c_{4}} \tilde{\alpha}_{2} \dot{\hat{\alpha}}_{2} \\
& +3 \kappa\left(k_{5}+k_{7}+\widehat{D}_{r} \mu_{1}+\widehat{D}_{t} \mu_{2}+\widehat{\alpha}_{1} \varsigma_{1} \mu_{3}+\widehat{\alpha}_{2} \varsigma_{2} \mu_{4}\right) .
\end{aligned}
$$

Combining the control laws defined in Eqs. (31)-(34), the following expression can be obtained:

$$
\begin{aligned}
& \dot{V}_{1} \leq \tilde{\alpha}_{1} \varsigma_{1}\left\|\mathbf{s}_{1}\right\|+\tilde{\alpha}_{2} \varsigma_{2}\left\|\mathbf{s}_{2}\right\|+\left\|\mathbf{s}_{1}\right\| \tilde{D}_{r}+\left\|\mathbf{s}_{2}\right\| \tilde{D}_{t}-k_{6}\left\|\mathbf{s}_{1}\right\|^{2} \\
& -k_{8}\left\|\mathbf{s}_{2}\right\|^{2}-\tilde{D}_{r}\left(\left\|\mathbf{s}_{1}\right\| \tanh \left(\frac{\left\|\mathbf{s}_{1}\right\|}{3 \mu_{1}}\right)-c_{5} \widehat{D}_{r}\right) \\
& -\tilde{D}_{t}\left(\left\|\mathbf{s}_{2}\right\| \tanh \left(\frac{\left\|\mathbf{s}_{2}\right\|}{3 \mu_{2}}\right)-c_{6} \widehat{D}_{t}\right) \\
& -\tilde{\alpha}_{1}\left(c_{1}\left\|\mathbf{s}_{1}\right\| \tanh \left(\frac{\varsigma_{1}\left\|\mathbf{s}_{1}\right\|}{3 \mu_{3}}\right)-c_{7} \widehat{\alpha}_{1}\right) \\
& -\tilde{\alpha}_{2}\left(\varsigma_{2}\left\|\mathbf{s}_{2}\right\| \tanh \left(\frac{\varsigma_{2}\left\|\mathbf{s}_{2}\right\|}{3 \mu_{4}}\right)-c_{8} \widehat{\alpha}_{2}\right) \\
& +3 \kappa\left(k_{5}+k_{7}+\widehat{D}_{r} \mu_{1}+\widehat{D}_{t} \mu_{2}+\widehat{\alpha}_{1} \varsigma_{1} \mu_{3}+\widehat{\alpha}_{2} \varsigma_{2} \mu_{4}\right) \\
& =\tilde{\alpha}_{1} \varsigma_{1}\left\|\mathbf{s}_{1}\right\|+\tilde{\alpha}_{2} \varsigma_{2}\left\|\mathbf{s}_{2}\right\|+\left\|\mathbf{s}_{1}\right\| \tilde{D}_{r}+\left\|\mathbf{s}_{2}\right\| \tilde{D}_{t}-k_{6}\left\|\mathbf{s}_{1}\right\|^{2} \\
& -k_{8}\left\|\mathbf{s}_{2}\right\|^{2}-\tilde{D}_{r}\left\|\mathbf{s}_{1}\right\| \tanh \left(\frac{\left\|\mathbf{s}_{1}\right\|}{3 \mu_{1}}\right) \\
& -\tilde{D}_{t}\left\|\mathbf{s}_{2}\right\| \tanh \left(\frac{\left\|\mathbf{s}_{2}\right\|}{3 \mu_{2}}\right)+c_{5} \tilde{D}_{r} \widehat{D}_{r}+c_{6} \tilde{D}_{t} \widehat{D}_{t} \\
& -\tilde{\alpha}_{1} \varsigma_{1}\left\|\mathbf{s}_{1}\right\| \tanh \left(\frac{\varsigma_{1}\left\|\mathbf{s}_{1}\right\|}{3 \mu_{3}}\right)-\tilde{\alpha}_{2} \varsigma_{2}\left\|\mathbf{s}_{2}\right\| \tanh \left(\frac{\varsigma_{2}\left\|\mathbf{s}_{2}\right\|}{3 \mu_{4}}\right) \\
& +c_{7} \tilde{\alpha}_{1} \widehat{\alpha}_{1}+c_{8} \tilde{\alpha}_{2} \widehat{\alpha}_{2}+3 \kappa\left(k_{5}+k_{7}\right)+\kappa \widehat{D}_{r} \mu_{1}+\kappa \widehat{D}_{t} \mu_{2} \\
& +3 \kappa \widehat{\alpha}_{1} \varsigma_{1} \mu_{3}+3 \kappa \widehat{\alpha}_{2} \varsigma_{2} \mu_{4} \leq \tilde{\alpha}_{1} \varsigma_{1}\left\|\mathbf{s}_{1}\right\|+\tilde{\alpha}_{2} \varsigma_{2}\left\|\mathbf{s}_{2}\right\| \\
& +\left\|\mathbf{s}_{1}\right\| \tilde{D}_{r}+\left\|\mathbf{s}_{2}\right\| \tilde{D}_{t}-k_{6}\left\|\mathbf{s}_{1}\right\|^{2}-k_{8}\left\|\mathbf{s}_{2}\right\|^{2}-\tilde{D}_{r}\left\|\mathbf{s}_{1}\right\| \\
& +3 \tilde{D}_{r} \mu_{1} \kappa-\tilde{D}_{t}\left\|\mathbf{s}_{2}\right\|+3 \tilde{D}_{t} \mu_{2} \kappa-\tilde{\alpha}_{1} \varsigma_{1}\left\|\mathbf{s}_{1}\right\|+3 \tilde{\alpha}_{1} \varsigma_{1} \mu_{3} \kappa \\
& -\tilde{\alpha}_{2} \varsigma_{2}\left\|\mathbf{s}_{2}\right\|+3 \tilde{\alpha}_{2} \varsigma_{2} \mu_{4} \kappa+c_{5} \tilde{D}_{r} \widehat{D}_{r}+c_{6} \tilde{D}_{t} \widehat{D}_{t}+c_{7} \tilde{\alpha}_{1} \widehat{\alpha}_{1} \\
& +c_{8} \tilde{\alpha}_{2} \widehat{\alpha}_{2}+3 \kappa\left(k_{5}+k_{7}+\widehat{D}_{r} \mu_{1}+\widehat{D}_{t} \mu_{2}+\widehat{\alpha}_{1} \varsigma_{1} \mu_{3}+\widehat{\alpha}_{2} \varsigma_{2} \mu_{4}\right) \\
& \leq-k_{6}\left\|\mathbf{s}_{1}\right\|^{2}-k_{8}\left\|\mathbf{s}_{2}\right\|^{2}+c_{5} \tilde{D}_{r} \widehat{D}_{r}+c_{6} \tilde{D}_{t} \widehat{D}_{t}+c_{7} \tilde{\alpha}_{1} \widehat{\alpha}_{1} \\
& +c_{8} \tilde{\alpha}_{2} \widehat{\alpha}_{2}+3 \kappa\left(k_{5}+k_{7}+D_{r} \mu_{1}+D_{t} \mu_{2}+\alpha_{1} \varsigma_{1} \mu_{3}+\alpha_{2} \varsigma_{2} \mu_{4}\right) \\
& \leq-k_{6}\left\|\mathbf{s}_{1}\right\|^{2}-k_{8}\left\|\mathbf{s}_{2}\right\|^{2}-\left(D_{r}-\widehat{D}_{r}\right)^{2}-\left(D_{t}-\widehat{D}_{t}\right)^{2} \\
& -\left(\alpha_{1}-\widehat{\alpha}_{1}\right)^{2}-\left(\alpha_{2}-\widehat{\alpha}_{2}\right)^{2}+\Delta_{1} \text {, }
\end{aligned}
$$


where $\Delta_{1}=\left(c_{5} D_{r}^{2} / 4\left(c_{5}-1\right)\right)+\left(c_{6} D_{t}^{2} / 4\left(c_{6}-1\right)\right)+\left(c_{7} \alpha_{1}^{2} / 4\left(c_{7}\right.\right.$ $-1))+\left(c_{8} \alpha_{2}^{2} / 4\left(c_{8}-1\right)\right)+3 \kappa\left(k_{5}+k_{7}+D_{r} \mu_{1}+D_{t} \mu_{2}+\alpha_{1} \varsigma_{1} \mu_{3}\right.$ $\left.+\alpha_{2} \varsigma_{2} \mu_{4}\right)$..

Equation (44) can be further rewritten as follows:

$$
\begin{aligned}
\dot{V}_{1} \leq & -\frac{2 k_{6}}{\lambda_{\max }(\mathbf{J})}\left(\frac{1}{2} \mathbf{s}_{1}^{\mathrm{T}} \mathbf{J} \mathbf{s}_{1}\right)-\frac{2 k_{8}}{m}\left(\frac{1}{2} \mathbf{s}_{2}^{\mathrm{T}} m \mathbf{s}_{2}\right)-2 c_{1}\left(\frac{1}{2 c_{1}} \tilde{D}_{r}^{2}\right) \\
& -2 c_{2}\left(\frac{1}{2 c_{2}} \tilde{D}_{t}^{2}\right)-2 c_{3}\left(\frac{1}{2 c_{3}} \alpha_{1}^{2}\right)-2 c_{4}\left(\frac{1}{2 c_{4}} \alpha_{2}^{2}\right)+\Delta_{1} \\
\leq & -\rho_{1} V_{1}+\Delta_{1},
\end{aligned}
$$

where $\rho_{1}=\min \left\{\left(2 k_{6} / \lambda_{\max }(\mathbf{J})\right),\left(2 k_{8} / m\right), 2 c_{1}, 2 c_{2}, 2 c_{3}, 2 c_{4}\right\}$.

Consequently, according to the aforementioned equation, we conclude that $\mathbf{s}_{1}, \mathbf{s}_{2}, \tilde{D}_{r}, \tilde{D}_{t}, \tilde{\alpha}_{1}$, and $\tilde{\alpha}_{2}$ exponentially converge to a bounded region with respect to $\Delta_{1}$. Then, the positive constants $\bar{D}_{r}, \bar{D}_{t}, \bar{\alpha}_{1}$, and $\bar{\alpha}_{2}$ that satisfy the $\bar{D}_{r} \geq D_{r}$, $\bar{D}_{r} \geq \widehat{D}_{r}, \bar{D}_{t} \geq D_{t}, \bar{D}_{t} \geq \widehat{D}_{t}$ and $\bar{\alpha}_{1} \geq \alpha_{1}, \bar{\alpha}_{1} \geq \widehat{\alpha}_{1}, \bar{\alpha}_{2} \geq \alpha_{2}, \bar{\alpha}_{2} \geq$ $\widehat{\alpha}_{2}$ conditions must exist.

To illustrate the finite-time stability, the Lyapunov function is defined as follows:

$$
\begin{aligned}
V_{2}= & \frac{1}{2} \mathbf{s}_{1}^{\mathrm{T}} \mathbf{J} \mathbf{s}_{1}+\frac{1}{2} \mathbf{s}_{2}^{\mathrm{T}} m \mathbf{s}_{2}+\frac{1}{c_{1}}\left(\bar{D}_{r}-\widehat{D}_{r}\right)^{2}+\frac{1}{c_{2}}\left(\bar{D}_{t}-\widehat{D}_{t}\right)^{2} \\
& +\frac{1}{c_{3}}\left(\bar{\alpha}_{1}-\widehat{\alpha}_{1}\right)^{2}+\frac{1}{c_{4}}\left(\bar{\alpha}_{2}-\widehat{\alpha}_{2}\right)^{2} .
\end{aligned}
$$

Combining the aforementioned schemes, the derivative of $V_{2}$ satisfies the following expression:

$$
\begin{aligned}
& \dot{V}_{2}=\mathbf{s}_{1}^{\mathrm{T}} \mathbf{J} \dot{\mathbf{s}}_{1}+\mathbf{s}_{2}^{\mathrm{T}} m \dot{\mathbf{s}}_{2}-\frac{2}{c_{1}}\left(\bar{D}_{r}-\widehat{D}_{r}\right) \dot{\hat{D}}_{r} \\
& -\frac{2}{c_{2}}\left(\bar{D}_{t}-\widehat{D}_{t}\right) \dot{\hat{D}}_{t}-\frac{2}{c_{3}}\left(\bar{\alpha}_{1}-\widehat{\alpha}_{1}\right) \dot{\widehat{\alpha}}_{1} \\
& -\frac{2}{c_{4}}\left(\bar{\alpha}_{2}-\widehat{\alpha}_{2}\right) \dot{\widehat{\alpha}}_{2} \\
& =\mathbf{s}_{1}^{\mathrm{T}}\left[-\mathbf{C}_{r} \tilde{\boldsymbol{\omega}}-\mathbf{n}_{r}+\boldsymbol{\varepsilon}_{r}+\mathbf{d}_{r}\right. \\
& +\frac{1}{2} \boldsymbol{J}\left[k_{1}+k_{2}\left(1+\tanh ^{\mathrm{T}}\left(\tilde{\mathbf{q}}_{v}\right) \tanh \left(\tilde{\mathbf{q}}_{v}\right)\right)\right] \\
& \left.\cdot\left(\tilde{\mathbf{q}}_{v}{ }^{\times}+\tilde{q}_{0} \mathbf{I}_{3}\right) \tilde{\boldsymbol{\omega}}\right]+\mathbf{s}_{2}^{\mathrm{T}}\left[-m \mathbf{C}_{t} \tilde{\boldsymbol{v}}-m \mathbf{n}_{t}+\boldsymbol{\varepsilon}_{t}+\mathbf{d}_{t}\right. \\
& \left.+m\left[k_{3}+k_{4}\left(1+\tanh ^{\mathrm{T}}(\tilde{\mathbf{r}}) \tanh (\tilde{\mathbf{r}})\right)\right]\left(\tilde{\mathbf{v}}-\mathbf{C}_{t} \tilde{\mathbf{r}}\right)\right] \\
& -\frac{2}{c_{1}}\left(\bar{D}_{r}-\widehat{D}_{r}\right) \dot{\hat{D}}_{r}-\frac{2}{c_{2}}\left(\bar{D}_{t}-\widehat{D}_{t}\right) \dot{\hat{D}}_{t} \\
& -\frac{2}{c_{3}}\left(\bar{\alpha}_{1}-\widehat{\alpha}_{1}\right) \dot{\widehat{\alpha}}_{1}-\frac{2}{c_{4}}\left(\bar{\alpha}_{2}-\widehat{\alpha}_{2}\right) \dot{\widehat{\alpha}}_{2} \\
& \leq \alpha_{1} \varsigma_{1}\left\|\mathbf{s}_{1}\right\|+\alpha_{2} \varsigma_{2}\left\|\mathbf{s}_{2}\right\|+\mathbf{s}_{1}^{\mathrm{T}}\left(\boldsymbol{\varepsilon}_{r}+\mathbf{d}_{r}\right) \\
& +\mathbf{s}_{2}^{\mathrm{T}}\left(\boldsymbol{\varepsilon}_{t}+\mathbf{d}_{t}\right)-\frac{2}{c_{1}}\left(\bar{D}_{r}-\widehat{D}_{r}\right) \dot{\hat{D}}_{r} \\
& -\frac{2}{c_{2}}\left(\bar{D}_{t}-\widehat{D}_{t}\right) \dot{\hat{D}}_{t}-\frac{2}{c_{3}}\left(\bar{\alpha}_{1}-\widehat{\alpha}_{1}\right) \dot{\widehat{\alpha}}_{1} \\
& -\frac{2}{c_{4}}\left(\bar{\alpha}_{2}-\widehat{\alpha}_{2}\right) \dot{\widehat{\alpha}}_{2} \text {. }
\end{aligned}
$$

By using the proposed control scheme, the following expression can be obtained:$$
\dot{V}_{2} \leq \alpha_{1} \varsigma_{1}\left\|\mathbf{s}_{1}\right\|+\alpha_{2} \varsigma_{2}\left\|\mathbf{s}_{2}\right\|+\left\|\mathbf{s}_{1}\right\| D_{r}+\left\|\mathbf{s}_{2}\right\| D_{t}
$$$$
+\mathbf{s}_{1}^{\mathrm{T}}\left(-k_{5} \tanh \left(\mathbf{s}_{1}\right)-k_{6} \mathbf{s}_{1}-\widehat{D}_{r} \tanh \left(\frac{\mathbf{s}_{1}}{\mu_{1}}\right)\right.
$$$$
\left.-\widehat{\alpha}_{1} \varsigma_{1} \tanh \left(\frac{\varsigma_{1} \mathbf{s}_{1}}{\mu_{3}}\right)\right)+\mathbf{s}_{2}^{\mathrm{T}}\left(-k_{7} \tanh \left(\mathbf{s}_{2}\right)-k_{8} \mathbf{s}_{2}\right.
$$$$
\left.-\widehat{D}_{t} \tanh \left(\frac{\mathbf{s}_{2}}{\mu_{2}}\right)-\widehat{\alpha}_{2} \varsigma_{2} \tanh \left(\frac{\varsigma_{2} \mathbf{s}_{2}}{\mu_{4}}\right)\right)
$$$$
-\frac{2}{c_{1}}\left(\bar{D}_{r}-\widehat{D}_{r}\right) \dot{\hat{D}}_{r}-\frac{2}{c_{2}}\left(\bar{D}_{t}-\widehat{D}_{t}\right) \dot{\hat{D}}_{t}
$$$$
-\frac{2}{c_{3}}\left(\bar{\alpha}_{1}-\widehat{\alpha}_{1}\right) \dot{\hat{\alpha}}_{1}-\frac{2}{c_{4}}\left(\bar{\alpha}_{2}-\widehat{\alpha}_{2}\right) \dot{\hat{\alpha}}_{2}
$$$$
\leq \alpha_{1} \varsigma_{1}\left\|\mathbf{s}_{1}\right\|+\alpha_{2} \varsigma_{2}\left\|\mathbf{s}_{2}\right\|+\left\|\mathbf{s}_{1}\right\| D_{r}+\left\|\mathbf{s}_{2}\right\| D_{t}
$$$$
-k_{5}\left\|\mathbf{s}_{1}\right\|-k_{6}\left\|\mathbf{s}_{1}\right\|^{2}-\widehat{D}_{r} \mathbf{s}_{1}^{\mathrm{T}} \tanh \left(\frac{\mathbf{s}_{1}}{\mu_{1}}\right)
$$$$
-\widehat{\alpha}_{1} \varsigma_{1} \mathbf{s}_{1}^{\mathrm{T}} \tanh \left(\frac{\varsigma_{1} \mathbf{s}_{1}}{\mu_{3}}\right)-k_{7}\left\|\mathbf{s}_{2}\right\|-k_{8}\left\|\mathbf{s}_{2}\right\|^{2}
$$$$
-\widehat{D}_{t} \mathbf{s}_{2}^{\mathrm{T}} \tanh \left(\frac{\mathbf{s}_{2}}{\mu_{2}}\right)-\widehat{\alpha}_{2} \varsigma_{2} \mathbf{s}_{2}^{\mathrm{T}} \tanh \left(\frac{\varsigma_{2} \mathbf{s}_{2}}{\mu_{4}}\right)
$$$$
-\frac{2}{c_{1}}\left(\bar{D}_{r}-\widehat{D}_{r}\right) \dot{\hat{D}}_{r}-\frac{2}{c_{2}}\left(\bar{D}_{t}-\widehat{D}_{t}\right) \dot{\hat{D}}_{t}
$$$$
-\frac{2}{c_{3}}\left(\bar{\alpha}_{1}-\widehat{\alpha}_{1}\right) \dot{\hat{\alpha}}_{1}-\frac{2}{c_{4}}\left(\bar{\alpha}_{2}-\widehat{\alpha}_{2}\right) \dot{\hat{\alpha}}_{2}+3 \kappa\left(k_{5}+k_{7}\right)
$$$$
\leq \alpha_{1} \varsigma_{1}\left\|\mathbf{s}_{1}\right\|+\alpha_{2} \varsigma_{2}\left\|\mathbf{s}_{2}\right\|+\left\|\mathbf{s}_{1}\right\| D_{r}+\left\|\mathbf{s}_{2}\right\| D_{t}-k_{5}\left\|\mathbf{s}_{1}\right\|
$$$$
-k_{6}\left\|\mathbf{s}_{1}\right\|^{2}-\widehat{D}_{r}\left\|\mathbf{s}_{1}\right\|+3 \widehat{D}_{r} \mu_{1} \kappa-\widehat{\alpha}_{1} \varsigma_{1}\left\|\mathbf{s}_{1}\right\|
$$$$
+3 \widehat{\alpha}_{1} \varsigma_{1} \mu_{3} \kappa-k_{7}\left\|\mathbf{s}_{2}\right\|-k_{8}\left\|\mathbf{s}_{2}\right\|^{2}-\widehat{D}_{t}\left\|\mathbf{s}_{2}\right\|
$$$$
+3 \widehat{D}_{t} \mu_{2} \kappa-\widehat{\alpha}_{2} \varsigma_{2}\left\|\mathbf{s}_{2}\right\|+3 \widehat{\alpha}_{2} \varsigma_{2} \mu_{4} \kappa
$$$$
-\frac{2}{c_{1}}\left(\bar{D}_{r}-\widehat{D}_{r}\right) \dot{\hat{D}}_{r}-\frac{2}{c_{2}}\left(\bar{D}_{t}-\widehat{D}_{t}\right) \dot{\hat{D}}_{t}
$$$$
-\frac{2}{c_{3}}\left(\bar{\alpha}_{1}-\widehat{\alpha}_{1}\right) \dot{\hat{\alpha}}_{1}-\frac{2}{c_{4}}\left(\bar{\alpha}_{2}-\widehat{\alpha}_{2}\right) \dot{\hat{\alpha}}_{2}+3 \kappa\left(k_{5}+k_{7}\right)
$$$$
\leq-k_{5}\left\|\mathbf{s}_{1}\right\|-k_{7}\left\|\mathbf{s}_{2}\right\|+\varsigma_{1}\left\|\mathbf{s}_{1}\right\|\left(\bar{\alpha}_{1}-\widehat{\alpha}_{1}\right)
$$$$
+\varsigma_{2}\left\|\mathbf{s}_{2}\right\|\left(\bar{\alpha}_{2}-\widehat{\alpha}_{2}\right)+\left\|\mathbf{s}_{1}\right\|\left(\bar{D}_{r}-\widehat{D}_{r}\right)
$$$$
+\left\|\mathbf{s}_{2}\right\|\left(\bar{D}_{t}-\widehat{D}_{t}\right)-\frac{2}{c_{1}}\left(\bar{D}_{r}-\widehat{D}_{r}\right) \dot{\hat{D}}_{r}
$$$$
-\frac{2}{c_{2}}\left(\bar{D}_{t}-\widehat{D}_{t}\right) \dot{\hat{D}}_{t}-\frac{2}{c_{3}}\left(\bar{\alpha}_{1}-\widehat{\alpha}_{1}\right) \dot{\hat{\alpha}}_{1}
$$$$
-\frac{2}{c_{4}}\left(\bar{\alpha}_{2}-\widehat{\alpha}_{2}\right) \dot{\hat{\alpha}}_{2}
$$$$
+3 \kappa\left(k_{5}+k_{7}+\widehat{D}_{r} \mu_{1}+\widehat{D}_{t} \mu_{2}+\widehat{\alpha}_{1} \varsigma_{1} \mu_{3}+\widehat{\alpha}_{2} \varsigma_{2} \mu_{4}\right) \text {. }
$$

According to the designed adaptive laws, the following expression can be obtained: 


$$
\begin{aligned}
\dot{V}_{2} \leq & -k_{5}\left\|\mathbf{s}_{1}\right\|-k_{7}\left\|\mathbf{s}_{2}\right\|+\varsigma_{1}\left\|\mathbf{s}_{1}\right\|\left(\bar{\alpha}_{1}-\widehat{\alpha}_{1}\right)+\varsigma_{2}\left\|\mathbf{s}_{2}\right\|\left(\bar{\alpha}_{2}-\widehat{\alpha}_{2}\right) \\
& +\left\|\mathbf{s}_{1}\right\|\left(\bar{D}_{r}-\widehat{D}_{r}\right)+\left\|\mathbf{s}_{2}\right\|\left(\bar{D}_{t}-\widehat{D}_{t}\right) \\
& -2\left(\bar{D}_{r}-\widehat{D}_{r}\right)\left(\left\|\mathbf{s}_{1}\right\| \tanh \left(\frac{\left\|\mathbf{s}_{1}\right\|}{3 \mu_{1}}\right)-c_{5} \widehat{D}_{r}\right) \\
& -2\left(\bar{D}_{t}-\widehat{D}_{t}\right)\left(\left\|\mathbf{s}_{2}\right\| \tanh \left(\frac{\left\|\mathbf{s}_{2}\right\|}{3 \mu_{2}}\right)-c_{6} \widehat{D}_{t}\right) \\
& -2\left(\bar{\alpha}_{1}-\widehat{\alpha}_{1}\right)\left(\varsigma_{1}\left\|\mathbf{s}_{1}\right\| \tanh \left(\frac{\varsigma_{1}\left\|\mathbf{s}_{1}\right\|}{3 \mu_{3}}\right)-c_{7} \widehat{\alpha}_{1}\right) \\
& -2\left(\bar{\alpha}_{2}-\widehat{\alpha}_{2}\right)\left(\varsigma_{2}\left\|\mathbf{s}_{2}\right\| \tanh \left(\frac{\varsigma_{2}\left\|\mathbf{s}_{2}\right\|}{3 \mu_{4}}\right)-c_{8} \widehat{\alpha}_{2}\right) \\
& +3 \kappa\left(k_{5}+k_{7}+\widehat{D}_{r} \mu_{1}+\widehat{D}_{t} \mu_{2}+\widehat{\alpha}_{1} \varsigma_{1} \mu_{3}+\widehat{\alpha}_{2} \varsigma_{2} \mu_{4}\right) \\
= & -k_{5}\left\|\mathbf{s}_{1}\right\|-k_{7}\left\|\mathbf{s}_{2}\right\|+\varsigma_{1}\left\|\mathbf{s}_{1}\right\|\left(\bar{\alpha}_{1}-\widehat{\alpha}_{1}\right)+\varsigma_{2}\left\|\mathbf{s}_{2}\right\|\left(\bar{\alpha}_{2}-\widehat{\alpha}_{2}\right) \\
& +\left\|\mathbf{s}_{1}\right\|\left(\bar{D}_{r}-\widehat{D}_{r}\right)+\left\|\mathbf{s}_{2}\right\|\left(\bar{D}_{t}-\widehat{D}_{t}\right) \\
& -2\left(\bar{D}_{r}-\widehat{D}_{r}\right)\left\|\mathbf{s}_{1}\right\| \tanh \left(\frac{\left\|\mathbf{s}_{1}\right\|}{3 \mu_{1}}\right) \\
& -2\left(\bar{D}_{t}-\widehat{D}_{t}\right)\left\|\mathbf{s}_{2}\right\| \tanh \left(\frac{\left\|\mathbf{s}_{2}\right\|}{3 \mu_{2}}\right) \\
& -2\left(\bar{\alpha}_{1}-\widehat{\alpha}_{1}\right) \varsigma_{1}\left\|\mathbf{s}_{1}\right\| \tanh \left(\frac{\varsigma_{1}\left\|\mathbf{s}_{1}\right\|}{3 \mu_{3}}\right) \\
& -2\left(\bar{\alpha}_{2}-\widehat{\alpha}_{2}\right) \varsigma_{2}\left\|\mathbf{s}_{2}\right\| \tanh \left(\frac{\varsigma_{2}\left\|\mathbf{s}_{2}\right\|}{3 \mu_{4}}\right) \\
& +2 c_{5} \widehat{D}_{r}\left(\bar{D}_{r}-\widehat{D}_{r}\right)+2 c_{6} \widehat{D}_{t}\left(\bar{D}_{t}-\widehat{D}_{t}\right) \\
& +2 c_{7} \widehat{\alpha}_{1}\left(\bar{\alpha}_{1}-\widehat{\alpha}_{1}\right)+2 c_{8} \widehat{\alpha}_{2}\left(\bar{\alpha}_{2}-\widehat{\alpha}_{2}\right) \\
& +3 \kappa\left(k_{5}+k_{7}+\widehat{D}_{r} \mu_{1}+\widehat{D}_{t} \mu_{2}+\widehat{\alpha}_{1} \varsigma_{1} \mu_{3}+\widehat{\alpha}_{2} \varsigma_{2} \mu_{4}\right) .
\end{aligned}
$$

According to Lemma 4, the following expression can be obtained:

$$
\begin{aligned}
\dot{V}_{2} \leq & -k_{5}\left\|\mathbf{s}_{1}\right\|-k_{7}\left\|\mathbf{s}_{2}\right\|+\varsigma_{1}\left\|\mathbf{s}_{1}\right\|\left(\bar{\alpha}_{1}-\widehat{\alpha}_{1}\right) \\
& +\varsigma_{2}\left\|\mathbf{s}_{2}\right\|\left(\bar{\alpha}_{2}-\widehat{\alpha}_{2}\right)+\left\|\mathbf{s}_{1}\right\|\left(\bar{D}_{r}-\widehat{D}_{r}\right)+\left\|\mathbf{s}_{2}\right\|\left(\bar{D}_{t}-\widehat{D}_{t}\right) \\
& -2\left(\bar{D}_{r}-\widehat{D}_{r}\right)\left\|\mathbf{s}_{1}\right\|+6 \mu_{1} \kappa\left(\bar{D}_{r}-\widehat{D}_{r}\right)-2\left(\bar{D}_{t}-\widehat{D}_{t}\right)\left\|\mathbf{s}_{2}\right\| \\
& +6 \mu_{2} \kappa\left(\bar{D}_{t}-\widehat{D}_{t}\right)-2\left(\bar{\alpha}_{1}-\widehat{\alpha}_{1}\right) \varsigma_{1}\left\|\mathbf{s}_{1}\right\|+6 \mu_{3} \varsigma_{1} \kappa\left(\bar{\alpha}_{1}-\widehat{\alpha}_{1}\right) \\
& -2\left(\bar{\alpha}_{2}-\widehat{\alpha}_{2}\right) \varsigma_{2}\left\|\mathbf{s}_{2}\right\|+6 \mu_{4} \varsigma_{2} \kappa\left(\bar{\alpha}_{2}-\widehat{\alpha}_{2}\right)+2 c_{5} \widehat{D}_{r}\left(\bar{D}_{r}-\widehat{D}_{r}\right) \\
& +2 c_{6} \widehat{D}_{t}\left(\bar{D}_{t}-\widehat{D}_{t}\right)+2 c_{7} \widehat{\alpha}_{1}\left(\bar{\alpha}_{1}-\widehat{\alpha}_{1}\right)+2 c_{8} \widehat{\alpha}_{2}\left(\bar{\alpha}_{2}-\widehat{\alpha}_{2}\right) \\
& +3 \kappa\left(k_{5}+k_{7}+\widehat{D}_{r} \mu_{1}+\widehat{D}_{t} \mu_{2}+\widehat{\alpha}_{1} \varsigma_{1} \mu_{3}+\widehat{\alpha}_{2} \varsigma_{2} \mu_{4}\right) \\
= & -k_{5}\left\|\mathbf{s}_{1}\right\|-k_{7}\left\|\mathbf{s}_{2}\right\|-\left(\left\|\mathbf{s}_{1}\right\|-3 \mu_{1} \kappa\right)\left(\bar{D}_{r}-\widehat{D}_{r}\right) \\
& -\left(\left\|\mathbf{s}_{2}\right\|-3 \mu_{2} \kappa\right)\left(\bar{D}_{t}-\widehat{D}_{t}\right)-\left(\varsigma_{1}\left\|\mathbf{s}_{1}\right\|-3 \mu_{3} \varsigma_{1} \kappa\right)\left(\bar{\alpha}_{1}-\widehat{\alpha}_{1}\right) \\
& -\left(\varsigma_{2}\left\|\mathbf{s}_{2}\right\|-3 \mu_{4} \varsigma_{2} \kappa\right)\left(\bar{\alpha}_{2}-\widehat{\alpha}_{2}\right)+2 c_{5} \widehat{D}_{r}\left(\bar{D}_{r}-\widehat{D}_{r}\right) \\
& +2 c_{6} \widehat{D}_{t}\left(\bar{D}_{t}-\widehat{D}_{t}\right)+2 c_{7} \widehat{\alpha}_{1}\left(\bar{\alpha}_{1}-\widehat{\alpha}_{1}\right)+2 c_{8} \widehat{\alpha}_{2}\left(\bar{\alpha}_{2}-\widehat{\alpha}_{2}\right) \\
& +3 \kappa\left(k_{5}+k_{7}+\bar{D}_{r} \mu_{1}+\bar{D}_{t} \mu_{2}+\bar{\alpha}_{1} \varsigma_{1} \mu_{3}+\bar{\alpha}_{2} \varsigma_{2} \mu_{4}\right) \\
= & -k_{5}\left\|\mathbf{s}_{1}\right\|-k_{7}\left\|\mathbf{s}_{2}\right\|-\left(\left\|\mathbf{s}_{1}\right\|-3 \mu_{1} \kappa\right)\left(\bar{D}_{r}-\widehat{D}_{r}\right) \\
& -\left(\left\|\mathbf{s}_{2}\right\|-3 \mu_{2} \kappa\right)\left(\bar{D}_{t}-\widehat{D}_{t}\right)-\left(\varsigma_{1}\left\|\mathbf{s}_{1}\right\|-3 \mu_{3} \varsigma_{1} \kappa\right)\left(\bar{\alpha}_{1}-\widehat{\alpha}_{1}\right) \\
& -\left(\varsigma_{2}\left\|\mathbf{s}_{2}\right\|-3 \mu_{4} \varsigma_{2} \kappa\right)\left(\bar{\alpha}_{2}-\widehat{\alpha}_{2}\right)+\Delta_{2},
\end{aligned}
$$

where $\Delta_{2}=\left(c_{5} / 2\right) \bar{D}_{r}^{2}+\left(c_{6} / 2\right) \bar{D}_{t}^{2}+\left(c_{7} / 2\right) \bar{\alpha}_{1}^{2}+\left(c_{8} / 2\right) \bar{\alpha}_{2}^{2}+3 \kappa$ $\left(k_{5}+k_{7}+\bar{D}_{r} \mu_{1}+\bar{D}_{t} \mu_{2}+\bar{\alpha}_{1} \varsigma_{1} \mu_{3}+\bar{\alpha}_{2} \varsigma_{2} \mu_{4}\right)$.

Equation (50) can be rewritten as follows:

$$
\begin{aligned}
\dot{V}_{2} \leq & -k_{5} \sqrt{\frac{2}{\lambda_{\max }(\mathbf{J})}}\left(\frac{1}{2} \mathbf{s}_{1}^{\mathrm{T}} \mathbf{J} \mathbf{s}_{1}\right)^{1 / 2}-k_{7} \sqrt{\frac{2}{m}}\left(\frac{1}{2} \mathbf{s}_{2}^{\mathrm{T}} m \mathbf{s}_{2}\right)^{1 / 2} \\
& -\sqrt{c_{1}}\left(\left\|\mathbf{s}_{1}\right\|-3 \mu_{1} \kappa\right)\left(\frac{1}{c_{1}}\left(\bar{D}_{r}-\widehat{D}_{r}\right)^{2}\right)^{1 / 2} \\
& -\sqrt{c_{2}}\left(\left\|\mathbf{s}_{2}\right\|-3 \mu_{2} \kappa\right)\left(\frac{1}{c_{2}}\left(\bar{D}_{t}-\widehat{D}_{t}\right)^{2}\right)^{1 / 2} \\
& -\sqrt{c_{3}}\left(\varsigma_{1}\left\|\mathbf{s}_{1}\right\|-3 \mu_{3} \varsigma_{1} \kappa\right)\left(\frac{1}{c_{3}}\left(\bar{\alpha}_{1}-\widehat{\alpha}_{1}\right)^{2}\right)^{1 / 2} \\
& -\sqrt{c_{4}}\left(\varsigma_{2}\left\|\mathbf{s}_{2}\right\|-3 \mu_{4} \varsigma_{2} \kappa\right)\left(\frac{1}{c_{4}}\left(\bar{\alpha}_{2}-\widehat{\alpha}_{2}\right)^{2}\right)^{1 / 2}+\Delta_{2} \\
\leq & -\rho_{2} \mathbf{V}_{2}^{1 / 2}+\Delta_{2},
\end{aligned}
$$

where $\rho_{2}=\min \left\{k_{5} \sqrt{2 / \lambda_{\max }(\mathbf{J})}, k_{7} \sqrt{2 / m}, \sqrt{c_{1}}\left(\left\|\mathbf{s}_{1}\right\|-3 \mu_{1} \kappa\right)\right.$, $\sqrt{c_{2}}\left(\left\|\mathbf{s}_{2}\right\|-3 \mu_{2} \kappa\right), \sqrt{c_{3}}\left(c_{1}\left\|\mathbf{s}_{1}\right\|-3 \mu_{3} \varsigma_{1} \kappa\right), \sqrt{c_{4}}\left(c_{2}\left\|\mathbf{s}_{2}\right\|-3 \mu_{4}\right.$ $\left.\left.\varsigma_{2} \kappa\right)\right\}$.

Consequently, $\mathbf{s}_{1}$ and $\mathbf{s}_{2}$ converge to two small regions $\Theta_{1}$ and $\Theta_{2}$ in finite time, where $\Theta_{1}$ and $\Theta_{2}$ are positive constants. The following equations can be obtained from the definition of $\mathbf{s}_{1}$ and $\mathbf{s}_{2}$ :

$$
\begin{aligned}
& \tilde{\omega}_{i}+k_{1} \tilde{q}_{v i}+k_{2} \tanh \left(\tilde{q}_{v i}\right)=\zeta_{i},\left|\zeta_{i}\right| \leq \Theta_{1} \quad i=1,2,3, \\
& \tilde{v}_{i}+k_{3} \tilde{r}_{i}+k_{4} \tanh \left(\tilde{r}_{i}\right)=\vartheta_{i},\left|\vartheta_{i}\right| \leq \Theta_{2} \quad i=1,2,3 .
\end{aligned}
$$

Thus, the following expressions can be obtained:

$$
\begin{array}{r}
\tilde{\omega}_{i}+\left(k_{1}-\frac{\zeta_{i}}{2 \tilde{q}_{v i}}\right) \tilde{q}_{v i}+\left(k_{2}-\frac{\zeta_{i}}{2 \tanh \left(\tilde{q}_{v i}\right)}\right) \tanh \left(\tilde{q}_{v i}\right)=0 \\
i=1,2,3, \\
\tilde{v}_{i}+\left(k_{3}-\frac{\vartheta_{i}}{2 \tilde{r}_{i}}\right) \tilde{r}_{i}+\left(k_{4}-\frac{\vartheta_{i}}{2 \tanh \left(\tilde{r}_{i}\right)}\right) \tanh \left(\tilde{r}_{i}\right)=0 \\
i=1,2,3 .
\end{array}
$$

Consequently, the relative dynamic system is stabilized in finite time under the conditions $k_{1}-\zeta_{i} / 2 \tilde{q}_{v i}>0, k_{2}-\zeta_{i} / 2$ $\tanh \left(\tilde{q}_{v i}\right)>0$, and $k_{3}-\vartheta_{i} / 2 \tilde{r}_{i}>0, k_{4}-\vartheta_{i} / 2 \tanh \left(\tilde{r}_{i}\right)>0$, which implies that the errors $\tilde{q}_{v i}$ and $\tilde{r}$ converge to the following region in finite time:

$$
\begin{aligned}
& \Delta_{\tilde{q}_{v}}=\max \left(\frac{\zeta_{i}}{2 k_{1}}, \frac{1}{2} \ln \left(\frac{k_{2}-\zeta_{i}}{k_{2}}\right)-\frac{1}{2}\right), \\
& \Delta_{\tilde{r}}=\max \left(\frac{\vartheta_{i}}{2 k_{3}}, \frac{1}{2} \ln \left(\frac{k_{4}-\vartheta_{i}}{k_{4}}\right)-\frac{1}{2}\right) .
\end{aligned}
$$

Furthermore, the relative angular velocity $\tilde{\omega}_{i}$ and relative velocity $\tilde{v}_{i}$ converge to the following regions in finite time: 


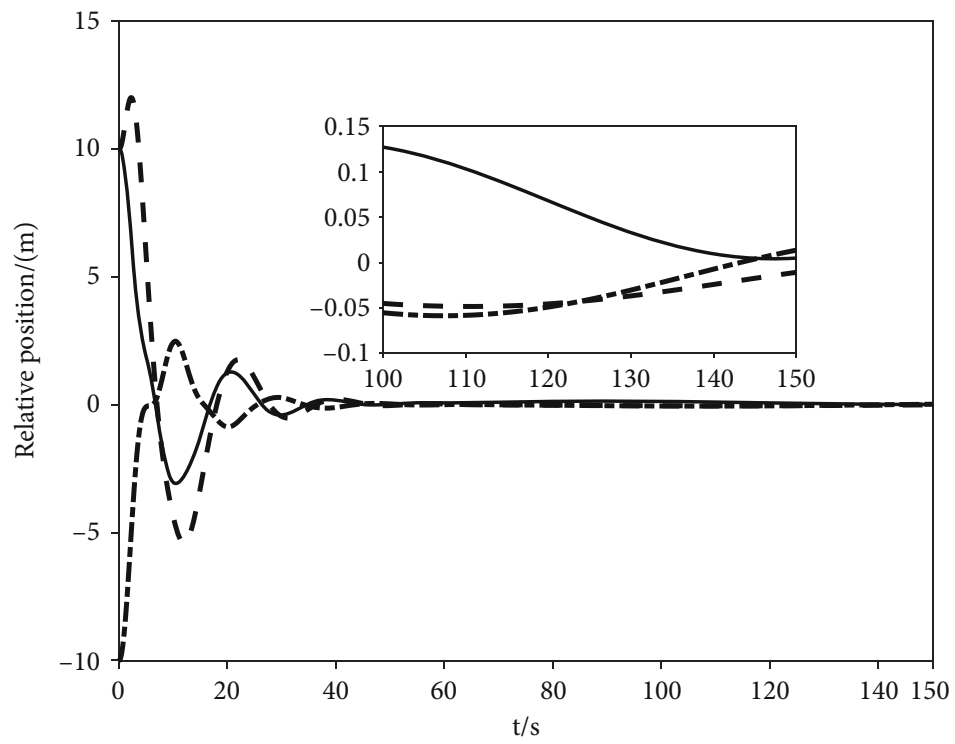

$-i=1$

- $-i=2$

--- $i=3$

Figure 1: Relative position.

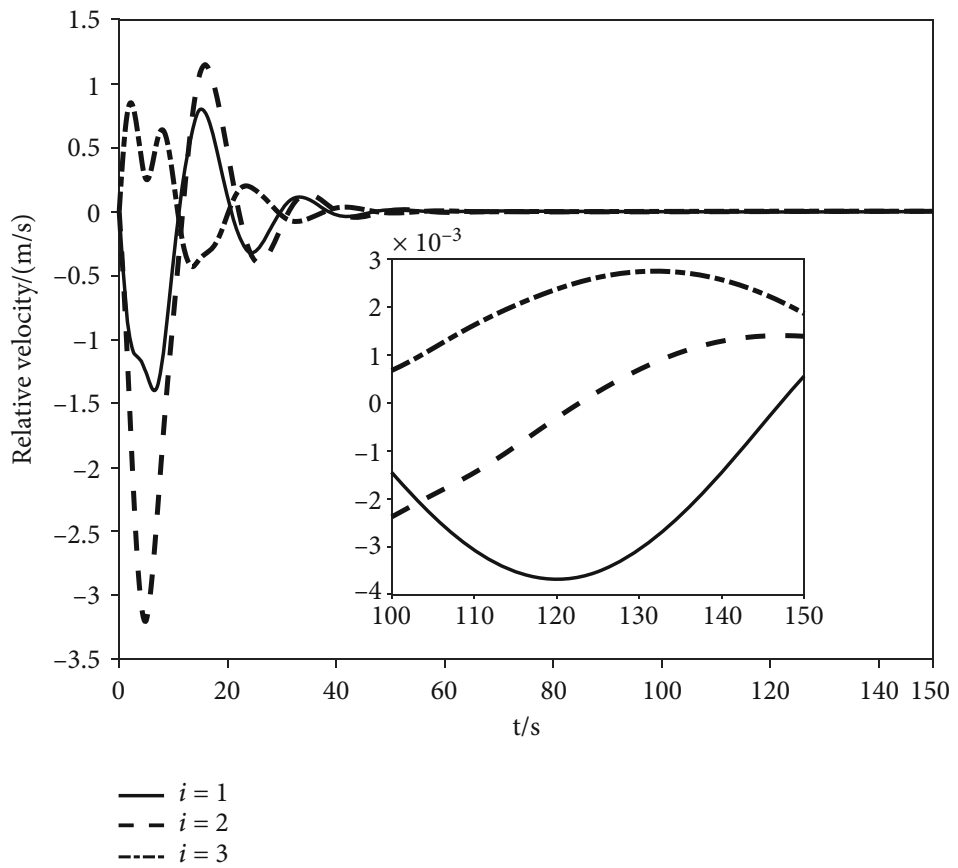

FIgURE 2: Relative velocity.

$$
\begin{aligned}
\left|\tilde{\omega}_{i}\right| & \leq\left|\zeta_{i}\right|+k_{1 i}\left|\tilde{q}_{v i}\right|+k_{2}\left|\tanh \left(\tilde{q}_{v i}\right)\right| \\
& \leq \Theta_{1}+k_{1} \Delta_{\tilde{q}_{v}}+k_{2} \tanh \left(\Delta_{\tilde{q}_{v}}\right)=\Theta_{q_{v}}, \\
\left|\tilde{v}_{i}\right| & \leq\left|\vartheta_{i}\right|+k_{3 i}\left|\tilde{r}_{i}\right|+k_{4 i}\left|\tanh \left(\tilde{r}_{i}\right)\right| \\
& \leq \Theta_{2}+\bar{k}_{3} \Delta_{\tilde{r}}+\bar{k}_{4} \tanh \left(\Delta_{\tilde{r}}\right)=\Theta_{r} .
\end{aligned}
$$

Therefore, Theorem 6 is proved.

\section{Simulation Results}

In this section, the effectiveness and advantage of the developed control strategy are presented. Detailed information regarding the orbit and these two spacecrafts is presented as follows [23]. In the simulation scenarios, a pursuer spacecraft is forced to rendezvous with the target spacecraft in an elliptical orbit with a perigee altitude $r_{p a}=400 \mathrm{~km}$ and 


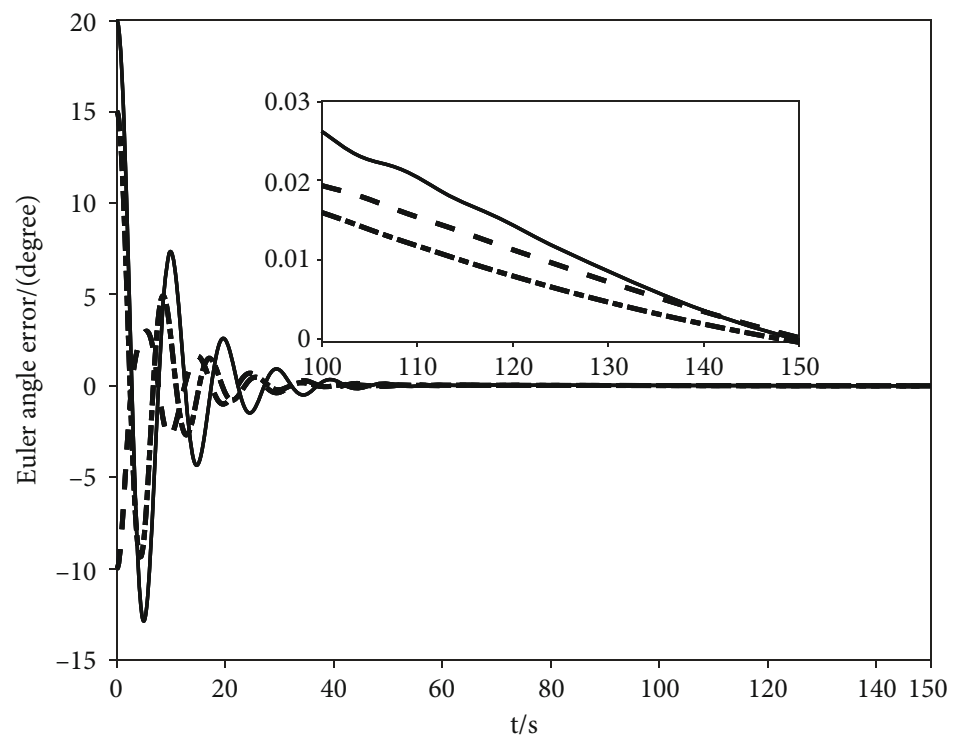

$-i=1$

- $-i=2$

--- $i=3$

FIgURE 3: Relative Euler angle.

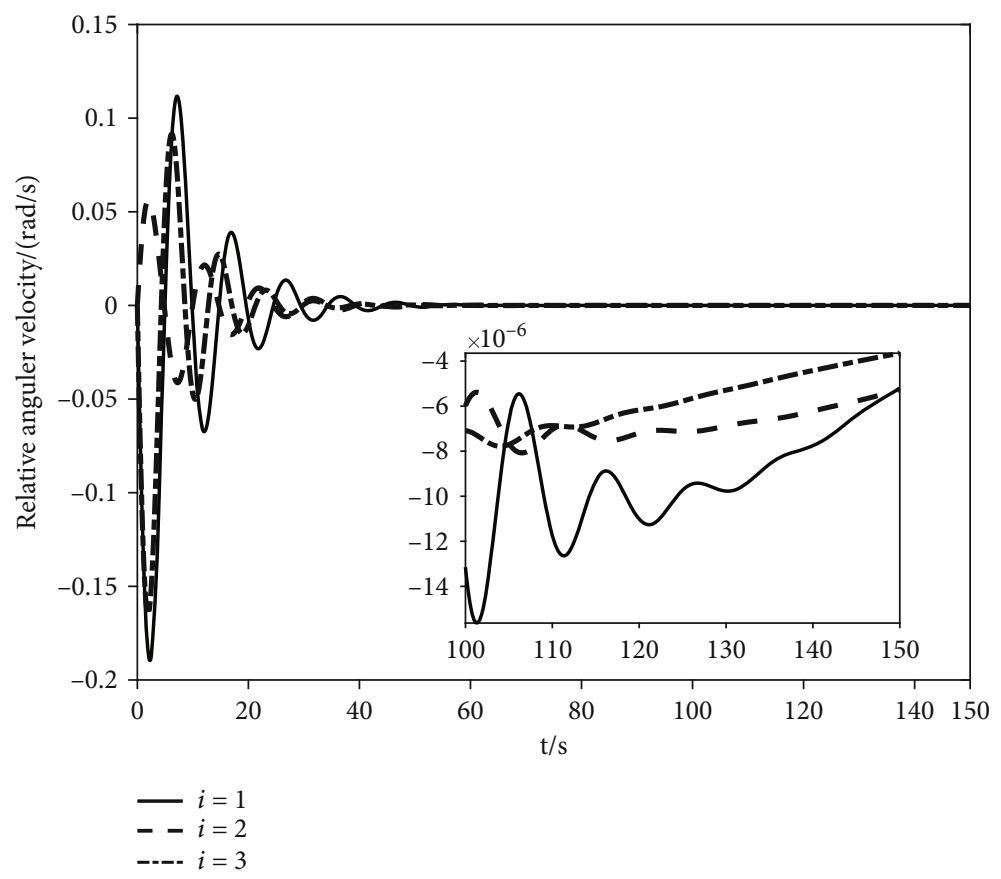

FIgURE 4: Relative angular velocity.

eccentricity $e=0.3$. The gravitational constant is $\mu=$ $3.986 \times 10^{14} \mathrm{Nm}^{2} / \mathrm{kg}$ and the radius of Earth is $R_{E}=$ $6371 \mathrm{~km}$. The mass and initial matrix of the purser spacecraft and the target spacecraft are chosen as $m=$ $90 \mathrm{~kg}, \mathbf{J}=\operatorname{diag}(20,20,15) \mathrm{kg} \cdot \mathrm{m}^{2}$ and $m_{t}=150 \mathrm{~kg}, \mathbf{J}_{t}=\operatorname{diag}$ $(26,16,21) \mathrm{kg} \cdot \mathrm{m}^{2}$, respectively. The initial true anomaly is $v(0)=10^{\circ}$.
The target spacecraft is supposed to service with the following position:

$$
\mathbf{r}_{t}=\left[r_{t}, 0,0\right]^{\mathrm{T}}, r_{t}=\frac{a\left(1-e^{2}\right)}{1+e \cos v},
$$




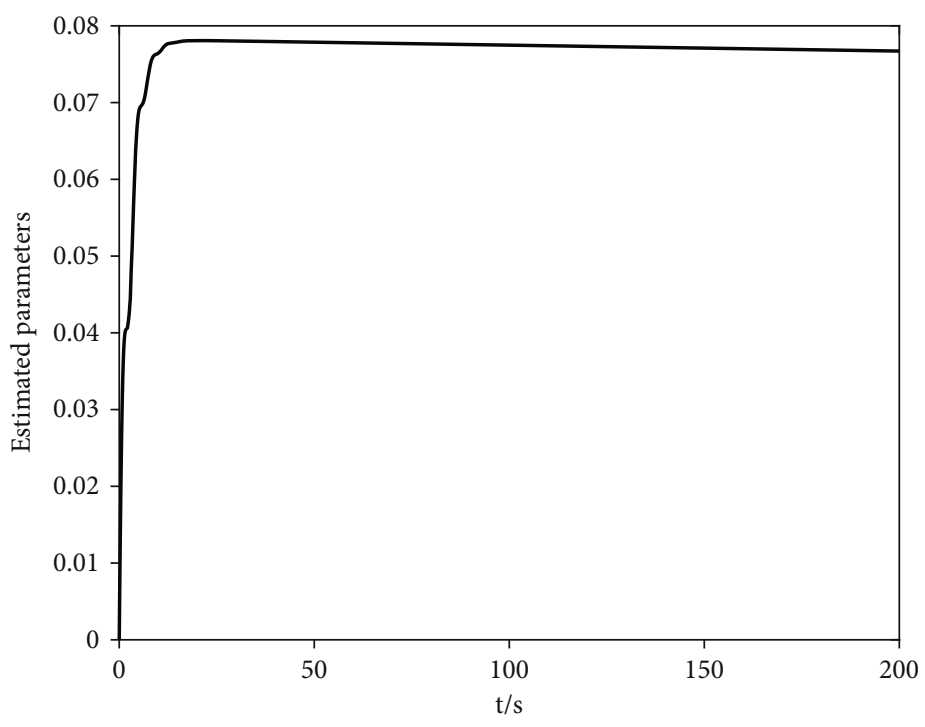

FIGURE 5: Estimation of $\beta_{r}$.

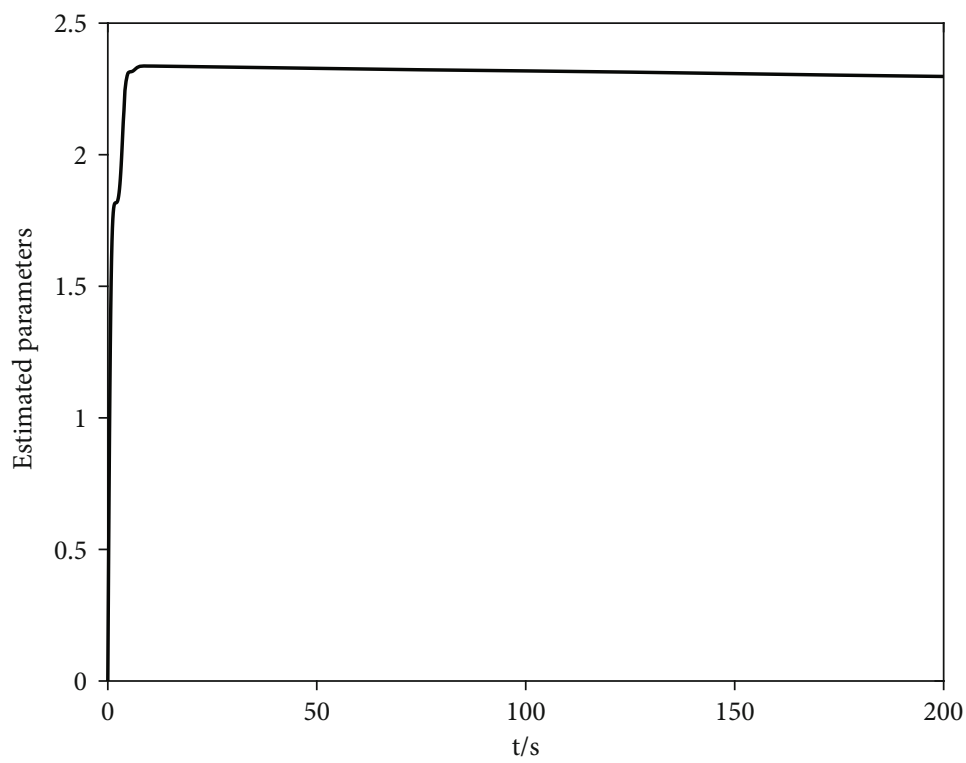

Figure 6: Estimation of $\beta_{t}$.

where $a=R_{E}+\left(r_{p a} / 1-e\right)$ denotes the semimajor axis. The parameter $v$ is expressed as follows:

$$
\dot{v}=\frac{n(1+e \cos v)^{2}}{\left(1-e^{2}\right)^{3 / 2}}, \ddot{v}=\frac{2 n^{2} e(1+e \cos v)^{3} \sin v}{\left(1-e^{2}\right)^{3}},
$$

where $n=\sqrt{u / a^{3}}$. For the pursuer spacecraft, its rendezvous position is expressed as $\boldsymbol{\delta}_{t}=[0,5,0]^{\mathrm{T}}$ in the body coordinate frame of the target. The angular velocity of the target and the external disturbances are expressed as follows:

$$
\begin{aligned}
\boldsymbol{\tau}_{d} & =0.02 \times\left(1+\cos \left(\frac{\pi}{150} t\right)+\sin \left(\frac{\pi}{150} t\right)\right)[1 ; 1 ; 1]^{\mathrm{T}} \mathrm{N} \cdot \mathrm{m}, \\
\mathbf{f}_{d} & =0.05 \times\left(1+\cos \left(\frac{\pi}{150} t\right)+\sin \left(\frac{\pi}{150} t\right)\right)[1 ; 1 ; 1]^{\mathrm{T}} \mathrm{N} \cdot \mathrm{m} .
\end{aligned}
$$

The initial states are set as follows: the initial Euler angle error is $\Theta(0)=[19.9984-9.998715 .0050]^{\mathrm{T}} \operatorname{deg}, \tilde{\boldsymbol{\omega}}=\left[\begin{array}{lll}0 & 0 & 0\end{array}\right]^{\mathrm{T}}$, $\tilde{\mathbf{r}}(0)=[10,10,-10]^{\mathrm{T}}$, and $\tilde{\mathbf{v}}(0)=\left[\begin{array}{ll}0 & 0\end{array}\right]^{\mathrm{T}}$.

The design parameters are set as follows: $k_{1}=2, k_{2}=2$, $k_{3}=0.5, k_{4}=0.1, k_{5}=0.05, k_{6}=4, k_{7}=10, k_{8}=10, c_{1}=0.02$, $c_{2}=0.001, c_{3}=0.05, c_{4}=0.001, c_{5}=1.1, c_{6}=5, c_{7}=1.1$, and 


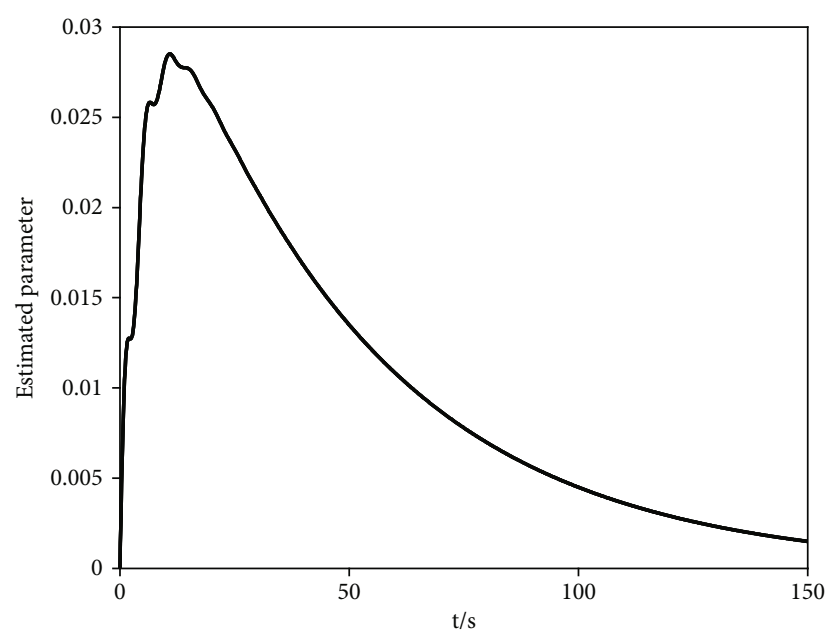

FIgURE 7: Estimation of $D_{r}$.

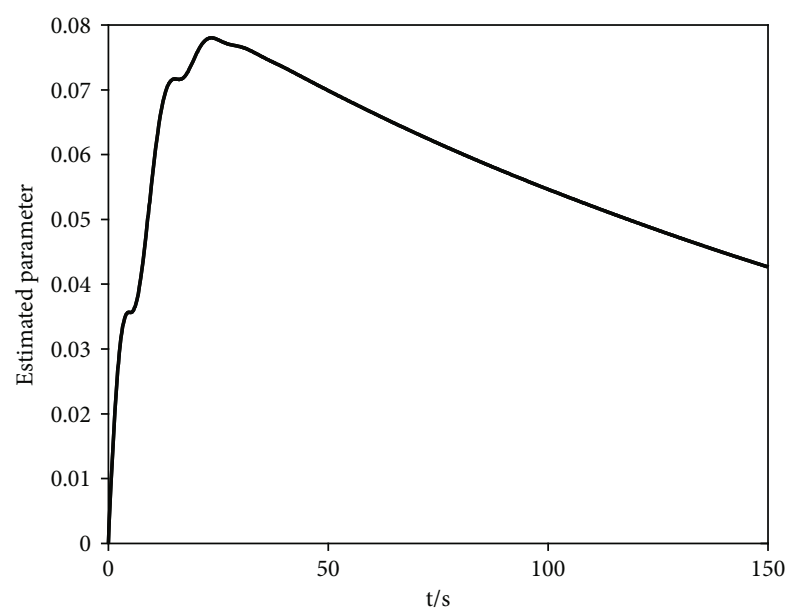

Figure 8: Estimation of $D_{t}$.

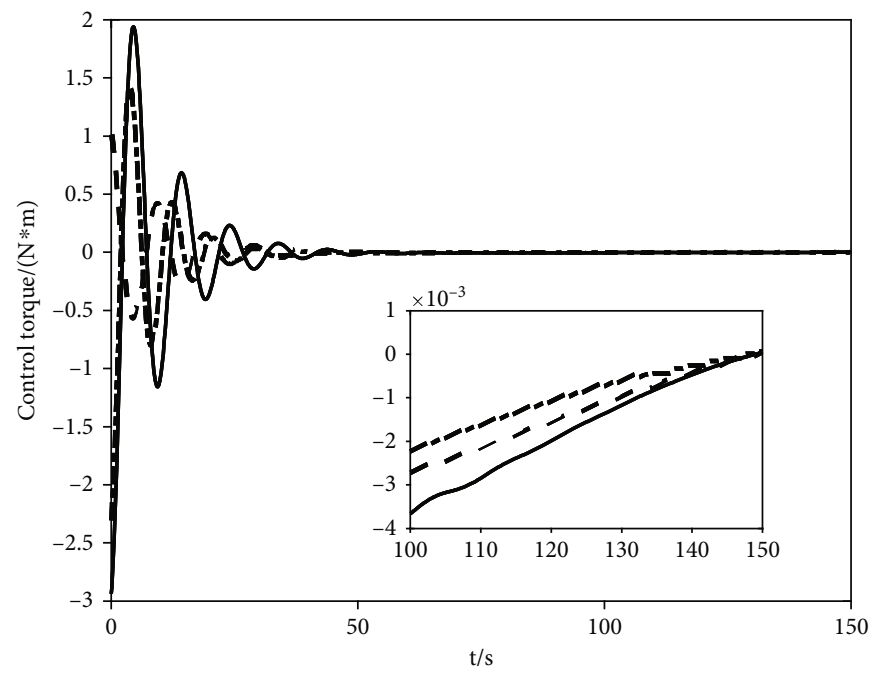

$\begin{aligned} i & =1 \\ --i & =2\end{aligned}$

--- $i=3$

FIGURE 9: Control torque $\boldsymbol{\tau}_{r}$. 


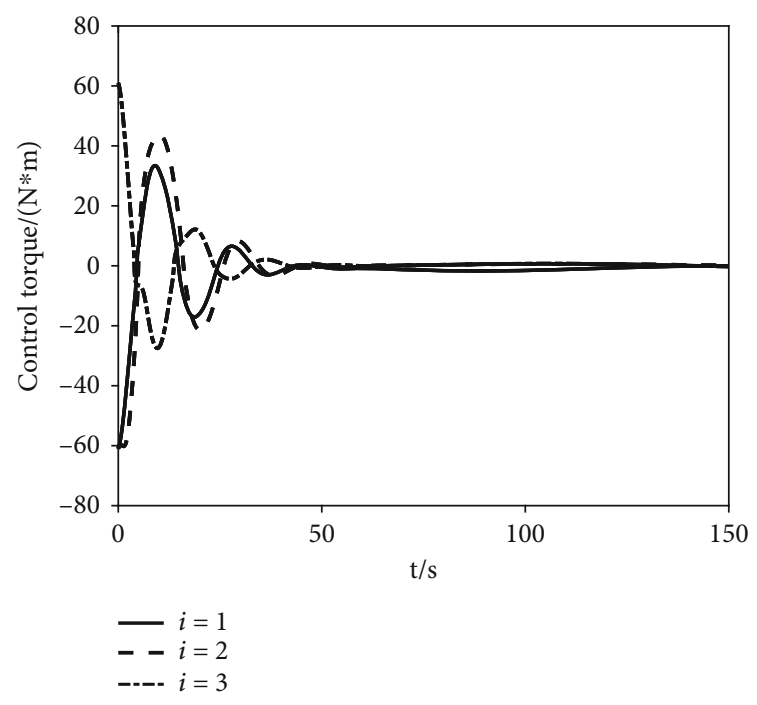

Figure 10: Control torque $\tau_{t}$.

$c_{8}=5$. The simulation results are illustrated in Figures 1-10. Figures 1-4 indicate that the relative attitude dynamics can be stabilized within $40 \mathrm{~s}$ and that the finite-time stability for the relative orbit dynamics can be ensured in $50 \mathrm{~s}$. Figures 5-8 present the estimated valuables, which have an upper bound under the proposed adaptive laws. Figures 9 and 10 display the control torques of the developed controllers. These simulation results indicate that the control objective can be achieved with satisfactory performance and that the chattering phenomenon does not occur in the controllers.

\section{Conclusion}

In this study, we focus on the finite-time model-free tracking control problem for the spacecraft rendezvous maneuver under external disturbances. By resorting to a well-defined sliding mode surface, finite-time convergence of the tracking errors is ensured, and all closed-loop signals are upper bounded. Adaptive laws are embedded into the control schemes such that external disturbances and system uncertainties could be compensated. Moreover, the chattering issue inherent in sliding mode technologies could be tackled based on the hyperbolic tangent function. Upon utilizing the proposed method, rendezvous maneuver could be accomplished with satisfactory performance even when system parameters remain inaccessible to designers. Theoretical analysis and simulation results have been presented to illustrate the effectiveness of the proposed method.

\section{Data Availability}

No data were used to support this study.

\section{Conflicts of Interest}

Authors declare that there are no conflicts of interest regarding publication of this article.

\section{References}

[1] Y. Guo, J. Guo, and S. Song, "Backstepping control for attitude tracking of the spacecraft under input saturation," Acta Astronautica, vol. 138, pp. 318-325, 2017.

[2] F. Wang, M. Hou, X. Cao, and G. Duan, "Event-triggered backstepping control for attitude stabilization of spacecraft," Journal of the Franklin Institute, vol. 356, no. 16, pp. 94749501, 2019.

[3] H. Yoon and P. Tsiotras, "Spacecraft adaptive attitude and power tracking with variable speed control moment gyroscopes," Journal of Guidance, Control, and Dynamics, vol. 25, no. 6, pp. 1081-1090, 2002.

[4] H. Wen, X. Yue, P. Li, and J. Yuan, "Fast spacecraft adaptive attitude tracking control through immersion and invariance design," Acta Astronautica, vol. 139, pp. 77-84, 2017.

[5] B. Huang, A. Li, Y. Guo, and C. Q. Wang, "Rotation matrix based finite-time attitude synchronization control for spacecraft with external disturbances," ISA Transactions, vol. 85, pp. 141-150, 2019.

[6] N. Zhou, Y. Xia, M. Wang, and M. Fu, "Finite-time attitude control of multiple rigid spacecraft using terminal sliding mode," International Journal of Robust and Nonlinear Control, vol. 25, no. 12, pp. 1862-1876, 2015.

[7] B. Huang, A. Li, Y. Guo, C. Q. Wang, and J. H. Guo, "Finitetime fault-tolerant attitude tracking control for spacecraft without unwinding," Proceedings of the Institution of Mechanical Engineers, Part G: Journal of Aerospace Engineering, vol. 233, no. 6, pp. 2119-2130, 2019.

[8] B. Huang, A. Li, Y. Guo, and C. Q. Wang, "Fixed-time attitude tracking control for spacecraft without unwinding," Acta Astronautica, vol. 151, pp. 818-827, 2018.

[9] K. Li and H. Ji, "Inverse optimal adaptive backstepping control for spacecraft rendezvous on elliptical orbits," International Journal of Control, vol. 91, no. 10, pp. 2303-2313, 2017.

[10] L. Sun, W. Huo, and Z. Jiao, "Adaptive backstepping control of spacecraft rendezvous and proximity operations with input saturation and full-state constraint," IEEE Transactions on Industrial Electronics, vol. 64, no. 1, pp. 480-492, 2016.

[11] X. Shao and Q. Hu, "Adaptive control for autonomous spacecraft rendezvous with approaching path constraint," in 2019 Chinese Control Conference (CCC), pp. 8188-8193, Guangzhou, China, July 2019.

[12] Y. Wang and H. Ji, "Integrated relative position and attitude control for spacecraft rendezvous with ISS and finite-time convergence," Aerospace Science and Technology, vol. 85, pp. 234$245,2019$.

[13] B. Jiang, Q. Hu, and M. I. Friswell, "Fixed-time rendezvous control of spacecraft with a tumbling target under loss of actuator effectiveness," IEEE Transactions on Aerospace and Electronic Systems, vol. 52, no. 4, pp. 1576-1586, 2016.

[14] K. Xia and W. Huo, "Adaptive fault-tolerant control for cooperative spacecraft rendezvous and docking," in 2017 American Control Conference (ACC), pp. 3747-3752, Seattle, WA, USA, May 2017.

[15] Q. Li, B. Zhang, J. Yuan, and H. Wang, "Potential function based robust safety control for spacecraft rendezvous and proximity operations under path constraint," Advances in Space Research, vol. 62, no. 9, pp. 2586-2598, 2018.

[16] Q. Li, J. Yuan, B. Zhang, and H. Wang, “Artificial potential field based robust adaptive control for spacecraft rendezvous 
and docking under motion constraint," ISA Transactions, vol. 95, pp. 173-184, 2019.

[17] Z. Zhu and Y. Guo, "Adaptive coordinated attitude control for spacecraft formation with saturating actuators and unknown inertia," Journal of the Franklin Institute, vol. 356, no. 2, pp. 1021-1037, 2019.

[18] J. Bae and Y. Kim, “Adaptive controller design for spacecraft formation flying using sliding mode controller and neural networks," Journal of the Franklin Institute, vol. 349, no. 2, pp. 578-603, 2012.

[19] L. Sun and W. Huo, "6-DOF integrated adaptive backstepping control for spacecraft proximity operations," IEEE Transactions on Aerospace and Electronic Systems, vol. 51, no. 3, pp. 2433-2443, 2015.

[20] N. Filipe and P. Tsiotras, "Adaptive position and attitudetracking controller for satellite proximity operations using dual quaternions," Journal of Guidance, Control, and Dynamics, vol. 38, no. 4, pp. 566-577, 2014.

[21] K. Xia and W. Huo, "Robust adaptive backstepping neural networks control for spacecraft rendezvous and docking with uncertainties," Nonlinear Dynamics, vol. 84, no. 3, pp. 16831695, 2016.

[22] D. Thakur, S. Srikant, and M. R. Akella, "Adaptive attitudetracking control of spacecraft with uncertain time-varying inertia parameters," Journal of Guidance, Control, and Dynamics, vol. 38, no. 1, pp. 41-52, 2014.

[23] K. Xia and S. Y. Park, "Adaptive control for spacecraft rendezvous subject to time-varying inertial parameters and actuator faults," Journal of Aerospace Engineering, vol. 32, no. 5, article 04019063, 2019.

[24] J. Zhang, J. D. Biggs, D. Ye, and Z. Sun, "Finite-time attitude set-point tracking for thrust-vectoring spacecraft rendezvous," Aerospace Science and Technology, vol. 96, article 105588, 2020.

[25] A. M. Zou, A. H. J. de Ruiter, and K. D. Kumar, "Distributed finite-time velocity-free attitude coordination control for spacecraft formations," Automatica, vol. 67, pp. 46-53, 2016.

[26] Y. Guo, B. Huang, S. Wang, A. J. Li, and C. Q. Wang, “Adaptive finite-time control for attitude tracking of spacecraft under input saturation," Journal of Aerospace Engineering, vol. 31, no. 2, article 04017086, 2018.

[27] G. Wu, S. M. Song, and J. Sun, "Finite-time antisaturation control for spacecraft rendezvous and docking with safe constraint," Proceedings of the Institution of Mechanical Engineers, Part G: Journal of Aerospace Engineering, vol. 233, no. 6, pp. 2170-2184, 2019.

[28] R. Xu, H. Ji, K. Li, Y. Kang, and K. Yang, "Relative position and attitude coupled control with finite-time convergence for spacecraft rendezvous and docking," in 2015 54th IEEE Conference on Decision and Control (CDC), pp. 3427-3432, Osaka, Japan, December 2015.

[29] C. Huang, Y. Wang, and X. Chen, "Finite-time attitude tracking control of air bearing table for spacecraft rendezvous and docking," Proceedings of the Institution of Mechanical Engineers, Part G: Journal of Aerospace Engineering, vol. 232, no. 1, pp. 96-110, 2016.

[30] M. J. Sidi, Spacecraft Dynamics and Control: A Practical Engineering Approach, Cambridge University Press, 2014. 